\title{
Peripheral immunophenotypes in children with multisystem inflammatory syndrome associated with SARS-CoV-2 infection
}

\author{
Michael J. Carter $\circledast^{1,2,7}$, Matthew Fish $\oplus^{3,4,5,7}$, Aislinn Jennings $\oplus^{3,4,7}$, Katie J. Doores ${ }^{4}$, \\ Paul Wellman $\oplus^{2}$, Jeffrey Seow ${ }^{4}$, Sam Acors ${ }^{\circledR}{ }^{4}$, Carl Graham ${ }^{4}$, Emma Timms ${ }^{5}$, Julia Kenny ${ }^{1,2}$,

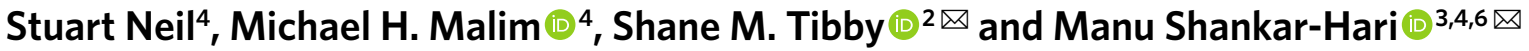

\begin{abstract}
Recent reports highlight a new clinical syndrome in children related to severe acute respiratory syndrome coronavirus 2 (SARS-CoV-2) - multisystem inflammatory syndrome in children (MIS-C)-which comprises multiorgan dysfunction and systemic inflammation ${ }^{2-13}$. We performed peripheral leukocyte phenotyping in 25 children with MIS-C, in the acute $(n=23$; worst illness within $72 \mathrm{~h}$ of admission), resolution $(n=14$; clinical improvement) and convalescent $(n=10$; first outpatient visit) phases of the illness and used samples from seven age-matched healthy controls for comparisons. Among the MIS-C cohort, 17 (68\%) children were SARS-CoV-2 seropositive, suggesting previous SARS-CoV-2 infections ${ }^{14,15}$, and these children had more severe disease. In the acute phase

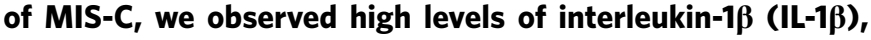
IL-6, IL-8, IL-10, IL-17, interferon- $\gamma$ and differential T and B cell subset lymphopenia. High CD64 expression on neutrophils and monocytes, and high HLA-DR expression on $\gamma \delta$ and $\mathrm{CD4}^{+}{ }^{+} \mathrm{CR} 7^{+} \mathrm{T}$ cells in the acute phase, suggested that these immune cell populations were activated. Antigen-presenting cells had low HLA-DR and CD86 expression, potentially indicative of impaired antigen presentation. These features normalized over the resolution and convalescence phases. Overall, MIS-C presents as an immunopathogenic illness ${ }^{1}$ and appears distinct from Kawasaki disease.
\end{abstract}

The study cohort, recruited during the pandemic, consisted of 25 patients (Extended Data Fig. 1) with pediatric multisystem inflammatory syndrome in children (MIS-C) temporally associated with severe acute respiratory syndrome coronavirus 2 (SARS-CoV-2) (also known as PIMS-TS ${ }^{16}$; Supplementary Tables 1 and 2). Table 1 summarizes the characteristics of the study cohort. The median (interquartile range (IQR)) age of our cohort was 12.5 years (7.7-14.4 years), of whom $40 \%(n=10)$ were girls, $40 \%$ $(n=10)$ were of white ethnicity, $72 \%(n=18)$ had gastrointestinal symptoms, $28 \%(n=7)$ had radiological evidence of pneumonia, $56 \%(n=14)$ had vasoactive infusions and $28 \%(n=7)$ had coronary artery dilatation or aneurysm (Montreal $Z$ score $\geq 2.0$; ref. ${ }^{17}$ ). Respiratory virus panels and bacterial infection screens were negative in all patients at hospitalization (Methods). Seventeen (68\%) patients were seropositive for SARS-CoV-2-specific antibodies, including one patient who was positive for SARS-CoV-2 by PCR with reverse transcription (RT-PCR). Among the eight (32.0\%) seronegative children, six had a clear history of either previous symptoms suggestive of SARS-CoV-2 infection, close household contact with confirmed cases of SARS-CoV-2 infection, presence at mass gatherings or parents who were health care workers. Seropositive status was associated with greater prevalence of gastrointestinal symptoms, worse left ventricular fractional shortening and increased use of immunomodulatory treatment. Coronary artery aneurysms were only observed in seropositive children. Previous MIS-C cohorts have not stratified clinical features and sequelae by seropositive status alone, so we were unable to make direct comparisons $\mathrm{s}^{2-13}$.

Overall, the clinical features of our cohort are comparable to those previously reported in the MIS-C literature ${ }^{2-13}$. In addition, our MIS-C cohort appears clinically distinct from Kawasaki disease, with only two patients within our MIS-C cohort meeting the criteria for complete Kawasaki disease (Table 1 and Extended Data Fig. 1c,d). The median age of our cohort (which was comparable to the median ages reported previously in the MIS-C literature ${ }^{5,7,9}$ ) was considerably older than that of Kawasaki disease, where the peak incidence is $1-3$ years of age ${ }^{18-21}$. Gastrointestinal symptoms and myocardial dysfunction are uncommon in Kawasaki disease, both of which were more prevalent in our seropositive MIS-C cases. In contrast with Kawasaki disease, MIS-C has not yet been described in Northeast Asian countries, such as Japan, despite the prevalence of SARS-CoV-2 in this region ${ }^{18,19}$ (Extended Data Fig. 1). The proportion of seroconverted patients in our cohort $(68 \%$; $95 \%$ confidence interval $=46.5-85.1 \%)$ was consistent with previous MIS-C reports $^{2,7,9-12,]}$. However, only one patient $(4 \%)$ in our cohort was positive for SARS-CoV-2 by RT-PCR, which is much lower than other MIS-C studies ${ }^{2,3,5-7,10,12,13}$. It is possible that the seronegative patients in our cohort were either never infected with SARS-CoV-2 or their antibodies declined rapidly following mild or asymptomatic infections ${ }^{22}$.

For further biological characterization of MIS-C, we outlined three clinically relevant illness phases: acute (T1; worst illness within $72 \mathrm{~h}$

'Department of Women and Children's Health, King's College London, London, UK. PPaediatric Intensive Care Unit, Evelina London Children's Hospital, London, UK. ${ }^{3}$ Department of Intensive Care Medicine, Guy's and St Thomas' NHS Foundation Trust, London, UK. 'Department of Infectious Diseases, School of Immunology and Microbial Sciences, King's College London, London, UK. 'Peter Gorer Department of Immunobiology, School of Immunology and Microbial Sciences, King's College London, London, UK. 'Present address: School of Immunology and Microbial Sciences, King's College London, London, UK. ${ }^{7}$ These authors contributed equally: Michael J. Carter, Matthew Fish, Aislinn Jennings. ${ }^{凶}$ e-mail: shane.tibby@gstt.nhs.uk;

manu.shankar-hari@kcl.ac.uk 
Table 1 | Characteristics of the study cohort

Characteristics

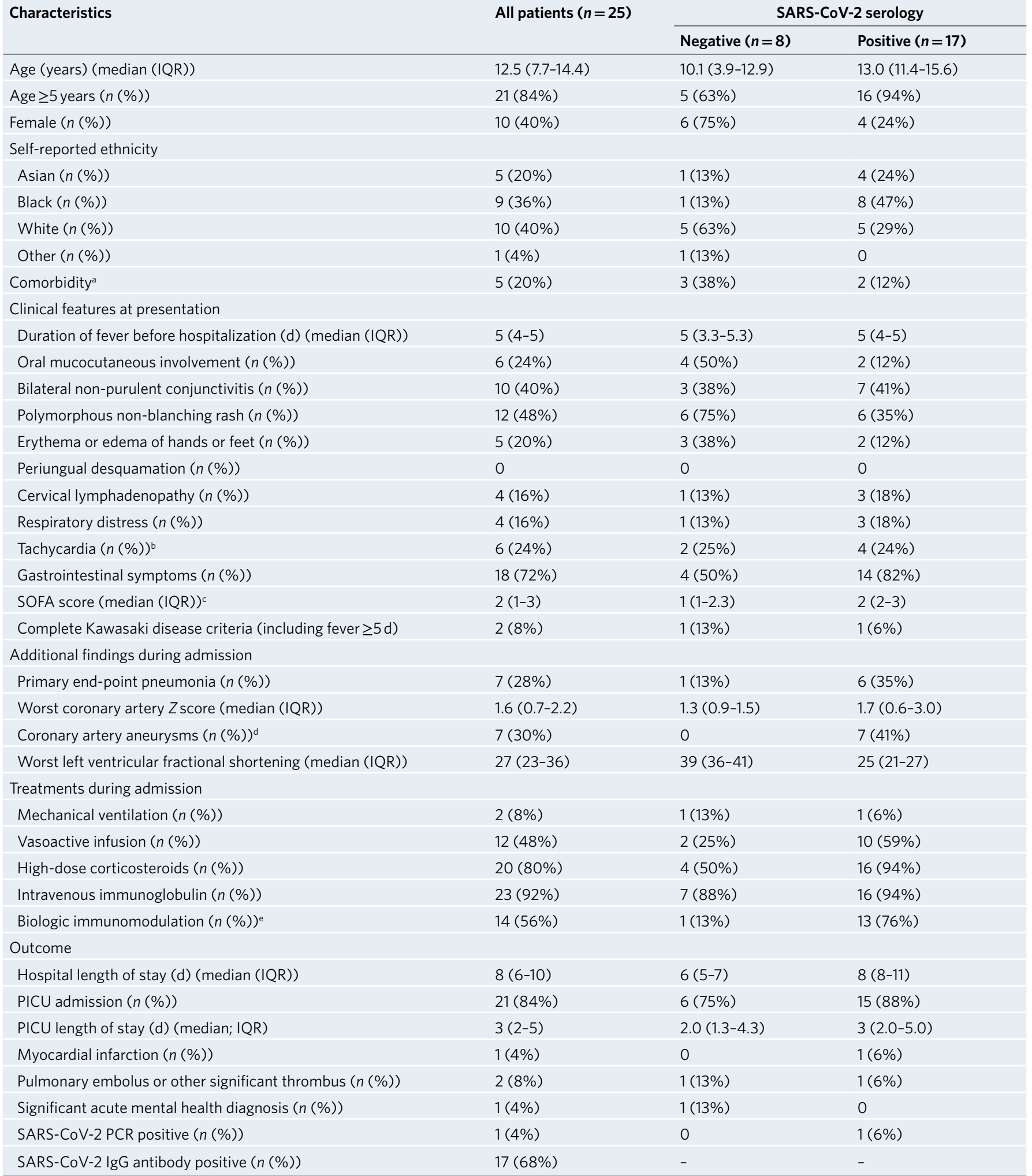

aTwo children had asthma (one with eczema and one with autistic spectrum disorder), one had a food allergy, one had hemoglobin C trait and one had aplastic anemia and immunosuppression (treated with ciclosporin). ${ }^{6}$ Tachycardia was determined using thresholds developed for the UK National Institute of Health and Clinical Excellence Guidelines for the Recognition and Management of Sepsis. 'Variables adjusted by age group (see Supplementary Table 2 for further details). ${ }^{\circledR}$ Montreal $Z$ score $\geq 2$ on echocardiography or computed tomography. ${ }^{e}$ One patient was treated with anakinra, four with infliximab and ten with tocilizumab. SOFA, sequential organ failure assessment.

of admission); resolution (T2; at clinical improvement, defined as improved respiratory status or cardiac support and C-reactive protein $(\mathrm{CRP})<100 \mathrm{mgl}^{-1}$ ); and convalescence (T3; at first outpatient follow-up after recovery). We monitored SARS-CoV-2 serology, biomarkers and immunophenotyped leukocytes at all three phases and select cytokines at $\mathrm{T} 1$. The timings of immunomodulatory treatments, 
which were prescribed agnostic of seroconversion status, are summarized in Fig. 1a.

In our cohort, immunoglobulin G (IgG) antibodies to SARS-Cov-2 nucleocapsid, the receptor-binding domain (RBD) of the spike protein and the spike protein itself were higher than their corresponding IgM titers, suggesting that SARS-CoV-2 infections had occurred $\geq 2$ weeks before clinical presentation ${ }^{14,15}$ (Fig. 1b). The median (IQR) median infectious dose $\left(\mathrm{ID}_{50}\right)$ for pseudoviral neutralization assay at $\mathrm{T} 1$ was 474 (355-644) units and positively correlated with RBD and spike protein IgG optical density values (Fig. 1c). There were no discernable trends in neutralizing antibody concentrations and the corresponding $\mathrm{ID}_{50}$ over the illness phases (Extended Data Fig. 2).

The acute phase was characterized by increased levels of cytokines, including interleukin-1 $\beta$ (IL-1 $\beta$ ), IL-6, IL-8, tumor necrosis factor- $\alpha$, IL-10, IL-17, interferon- $\gamma$ (IFN- $\gamma$ ) and IL-2 receptor agonist (Fig. 1d), with raised CRP (Fig. 1e) and ferritin, confirming acute inflammation. Raised amino (N)-terminal pro B-type natriuretic peptide (NT-proBNP; Fig. 1f) and troponin are indicative of myocardial dysfunction and injury, respectively ${ }^{12,13}$. Raised fibrinogen (Fig. 1g), raised D-dimer and low platelets in the acute phase suggest a procoagulant state. These changes normalized by convalescence. Although acute inflammation is common in Kawasaki disease, the procoagulant state seen in MIS-C patients is not a common feature of Kawasaki disease ${ }^{18-21}$ (Extended Data Fig. 3).

We immunophenotyped innate, $\mathrm{T}$ and $\mathrm{B}$ cells using three separate flow cytometry panels (Methods). For immunophenotyping, 23 patients had T1 samples, 14 had T2 samples and ten had T3 samples. Eighteen patients had matched samples between at least two phases (Fig. 1a). For comparison, we recruited seven children of similar age who were SARS-CoV-2 RT-PCR negative and/or serology negative and had been admitted for planned diagnostic or interventional procedures (Supplementary Table 3).

Absolute neutrophil, monocyte, dendritic cell and natural killer cell counts were similar during the acute, resolution and convalescence phases and were similar to counts in healthy controls (Fig. 2a-d). We considered the median fluorescence intensity (MFI) of cluster of differentiation 64 (CD64) as a neutrophil activation marker ${ }^{23}$. In the acute phase, the neutrophil CD64 MFI was notably increased compared with healthy controls but normalized by the resolution phase (Fig. 2e). The neutrophil activation inference was supported by high levels of IL- 8 . There was a concomitant decrease in the CD10 MFI on neutrophils, suggestive of a decrease in the frequency of mature neutrophils and potentially linked to the observed enhancement in T cell activation ${ }^{24,25}$ (Fig. 2f). Activated neutrophils could influence the functionality of T cells and $\mathrm{B}$ cells ${ }^{25}$.

In the acute phase, the monocyte CD14 (a Toll-like receptor 4 co-receptor ${ }^{26}$ ) MFI was reduced, while the CD64 MFI was increased (Extended Data Fig. 4), implying activation and cytokine production ${ }^{27,28}$. We used the human leukocyte antigen-DR isotype (HLA-DR) MFI on antigen-presenting cells (APCs; that is, monocytes, dendritic cells and B cells) and the CD86 MFI as surrogates of antigen presentation ability. We observed decreased HLA-DR and CD86 MFIs in the overall monocyte population (Fig. $2 \mathrm{~g}, \mathrm{~h}$ ) in the acute phase, although the classical monocyte proportions were unchanged (Extended Data Fig. 4). The frequency of conventional dendritic cells decreased in the resolution phase (Fig. 2i). The dendritic cells also had decreased HLA-DR and CD86 MFIs (Fig. 2j), which could be indicative of impaired antigen cross-presentation to $\mathrm{CD}^{+} \mathrm{T}$ cells and priming/polarizing of naive helper $\mathrm{T}$ cells ${ }^{29}$. There was a positive correlation between HLA-DR and CD86 MFIs in monocytes and dendritic cells (Fig. 2k,1).

We observed helper $\left(\mathrm{CD}^{+}\right)$, cytotoxic $\left(\mathrm{CD}^{+}\right)$and $\gamma \delta \mathrm{T}$ cell lymphopenia in the acute phase, which returned to normal by convalescence (Fig. $3 \mathrm{a}-\mathrm{d}$ ). There were notable differences in the magnitude of lymphopenia, the activation status and the time to normalization between $\mathrm{T}$ cell subsets (Fig. 3a-d). The HLA-DR MFI on $\mathrm{T}$ cells is considered indicative of activation ${ }^{30}$. Although the overall $\mathrm{CD}^{+}{ }^{+} \mathrm{T}$ cell HLA-DR MFI was similar to healthy controls, the $\mathrm{CD}^{+}{ }^{+} \mathrm{CCR}^{+} \mathrm{T}$ cells (mainly naive $\mathrm{T}$ cells and a small proportion of central memory T cells) had significantly higher HLA-DR MFIs during the acute phase, in contrast with the $\mathrm{CD} 4^{+} \mathrm{CCR} 7^{-}$effector T cell subsets and CD8 subsets (Fig. 3f,g and Extended Data Fig. 5). Consistent with the previous literature ${ }^{31}$, we observed higher proportions of $\mathrm{CD} 4^{+} \mathrm{CCR} 7^{+} \mathrm{T}$ cells in our cohort. $\gamma \delta \mathrm{T}$ cells, which have antiviral properties including IFN secretion, pathogen killing and cytotoxicity $^{32}$, were significantly reduced during the acute phase, with an increase in the HLA-DR MFI (Fig. 3h). There was a notable increase in regulatory $\mathrm{T}$ cell counts in the convalescence phase, but the proportion of activated regulatory $\mathrm{T}$ cells $\left(\mathrm{HLA}-\mathrm{DR}^{+}\right)^{30}$ with immunosuppressive effector functions ${ }^{33}$ was unchanged. There were no correlations between antibody titers and $\gamma \delta$ T cells or with $\mathrm{CD}^{+}{ }^{+} \mathrm{CCR}^{-}$effector T cells. Although the HLA-DR MFI within $\mathrm{CD}^{+} \mathrm{T}$ cells did not differ by CCR7 status, the HLA-DR MFIs on $\mathrm{CD}^{+} \mathrm{CCR}^{+}$and $\mathrm{CD}^{+}{ }^{+} \mathrm{CCR}^{+}$cells were positively correlated (Extended Data Fig. 5).

In the acute phase, total $\mathrm{B}$ cell counts and natural effector $\left(\mathrm{CD} 19^{+} \mathrm{CD} 27^{+} \operatorname{IgM}^{+} \mathrm{IgD}^{+}\right)$cells were notably reduced (Fig. 3i,j). Class-switched memory B cell counts $\left(\mathrm{CD} 19^{+} \mathrm{CD} 27^{+} \mathrm{IgM}^{-}\right.$cells) were higher in the resolution phase compared with the acute phase (Fig. 3k). The HLA-DR MFI was notably reduced in B cells in the acute phase (Fig. 31). The transitional cells were unchanged, with a progressive decrease in the proportion of $\mathrm{B}$ cells with the ability to produce low-affinity polyreactive antibodies $\left(\mathrm{CD}^{+} \mathrm{B}\right.$ cell subset $)^{34}$ and an increase in the proportions of plasmablasts $\left(\mathrm{CD} 19^{+} \mathrm{CD} 27^{+++} \mathrm{CD} 38^{+++}\right)$in some patients. The lack of correlations between spike protein-specific IgG titers and either plasmablasts or class-switched memory cells (Extended Data Fig. 5) has similarities to the COVID-19 immune responses in adults ${ }^{35,36}$. The significance and mechanisms underpinning these B cell changes in MIS- $C$ require further evaluation.

Fig. 1 | Cohort description, SARS-CoV-2 serology and biomarkers of inflammation, myocardial dysfunction and coagulation. a, Clinical time course of the patients recruited to the study. The place of care and clinical status of each child is indicated by color. Sampling phase and treatments administered are indicated by shapes. Patients are grouped vertically by serological status. IVIg, intravenous immunoglobulin. b, Correlation plots between IgG of nucleocapsid, RBD and spike protein and pseudoviral neutralization assays $\left(\mathrm{ID}_{50}\right.$ ). The regression line (solid) and $95 \% \mathrm{Cl}$ (gray shading) are shown. c. Serum cytokine concentrations in the acute phase ( $T 1 ; n=15$ samples), measured from samples taken before any immunomodulatory treatment. The gray bars represent the laboratory normal range. d, Optical density of IgM and IgG antibodies for nucleocapsid, RBD and spike. e-g, Serum CRP (e), serum NT-proBNP (f) and fibrinogen concentrations ( $\mathbf{g}$ ) during illness phases. For all box and whisker plots, the bottom border represents the 25 th percentile, the line bisecting the shaded region of the box represents the median, the upper border of the box represents the 75th percentile, the whiskers represent extreme values (1.5x the 75th (highest) and 25th (lowest) percentile values) and markers beyond the extreme line are outliers. Dots are colored by serology result (red: positive; blue: negative), with the red asterisk indicating the patient who was positive by RT-PCR for SARS-CoV-2. Further details on the clinical biomarkers ferritin, troponin, D-dimer and platelet counts are provided in Extended Data Figs. 1-3. A Spearman's rank test was used for the correlations. Significance testing between patients by phase of illness (T1 ( $n=25$ samples), T2 ( $n=15$ samples) and T3 ( $n=6$ samples)) was performed using two-sided Wilcoxon rank-sum tests with correction for multiple comparisons. 
We evaluated the clinical relevance of the illness phases we outlined earlier using principal component analysis and hierarchical clustering of the immunophenotyping markers we measured
(Extended Data Fig. 6). There were differences in the immunological features between illness phases. Innate and $\mathrm{T}$ cell activation features were dominant in the acute phase, but regulatory $\mathrm{T}$ cells

a

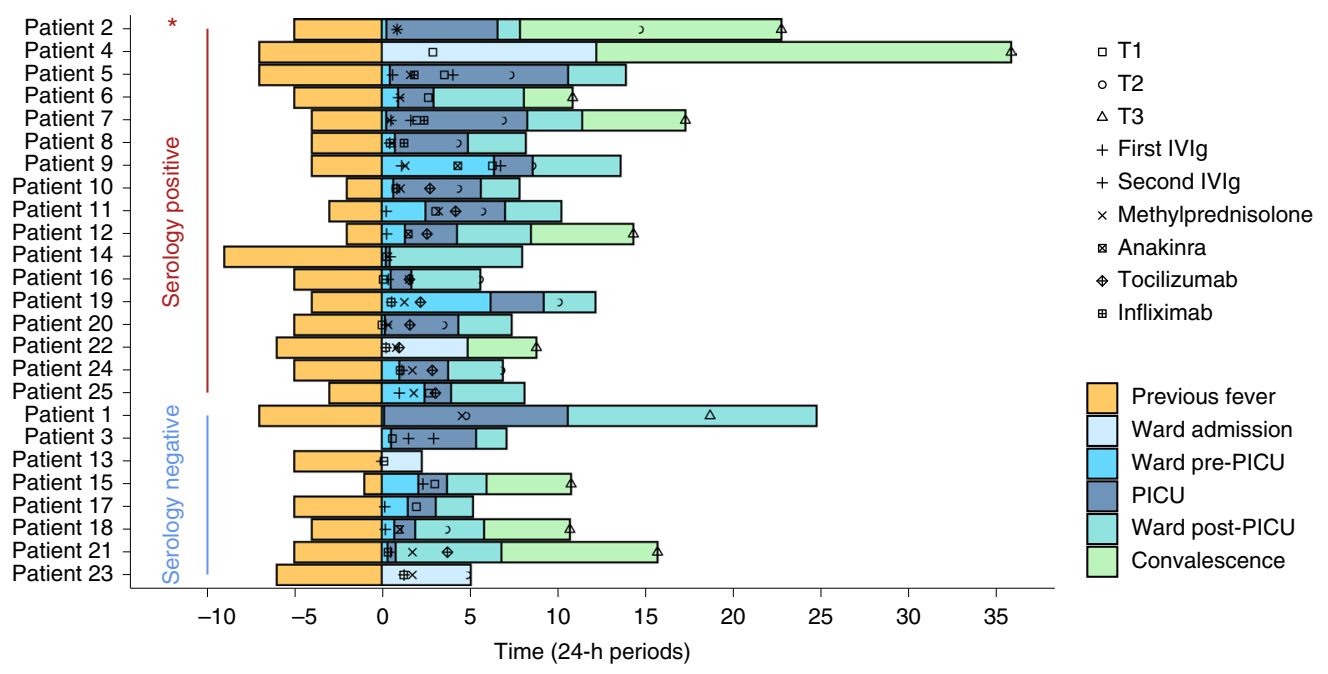

b

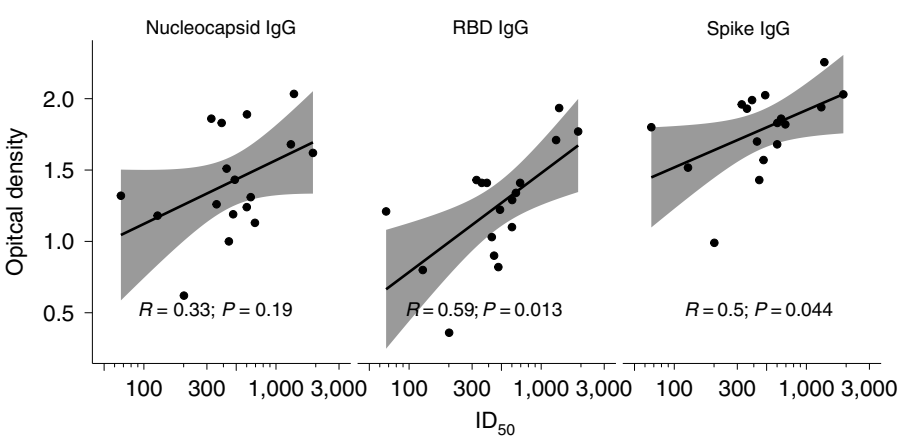

C

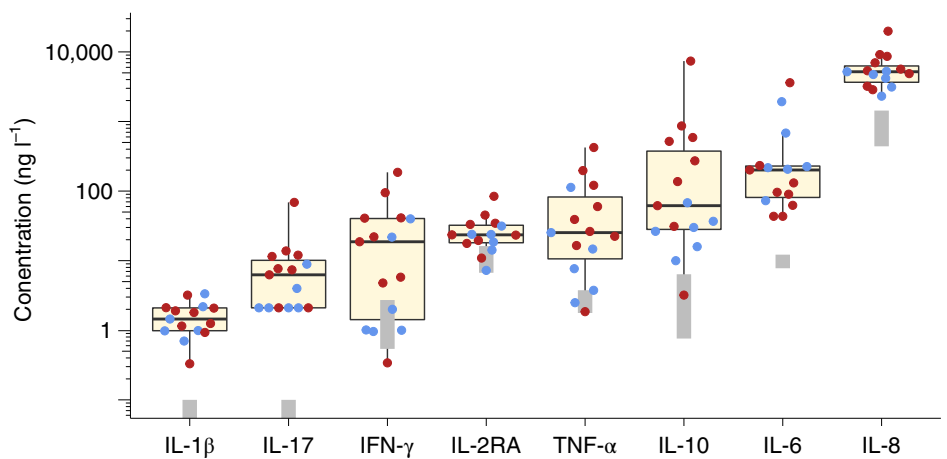

d

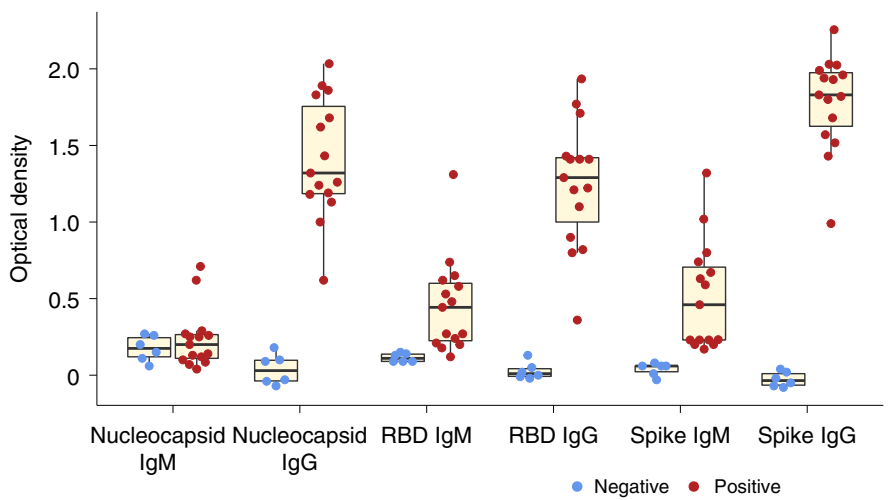

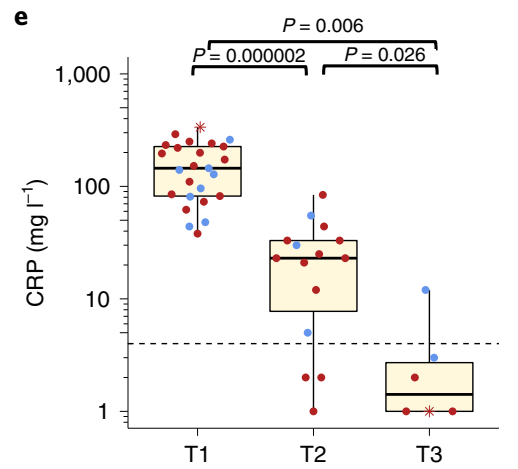

f

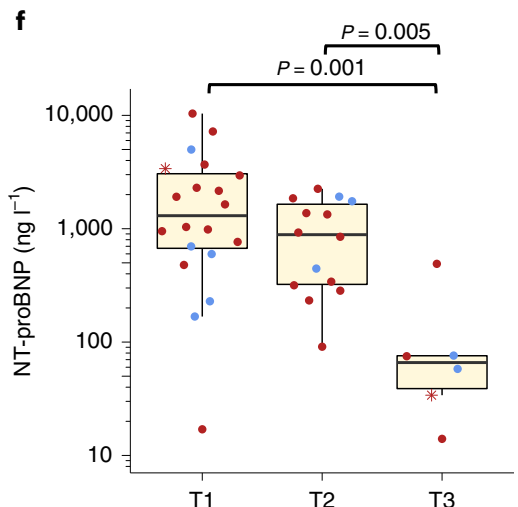

g

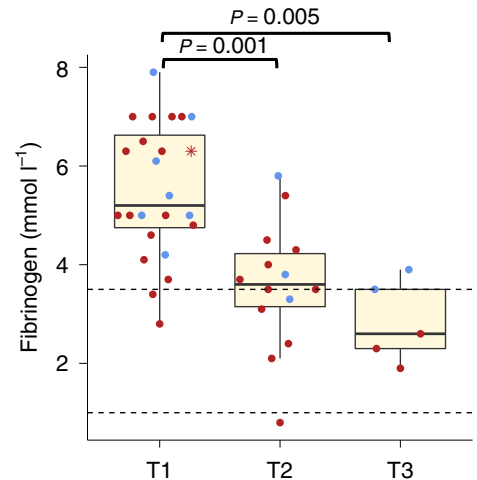



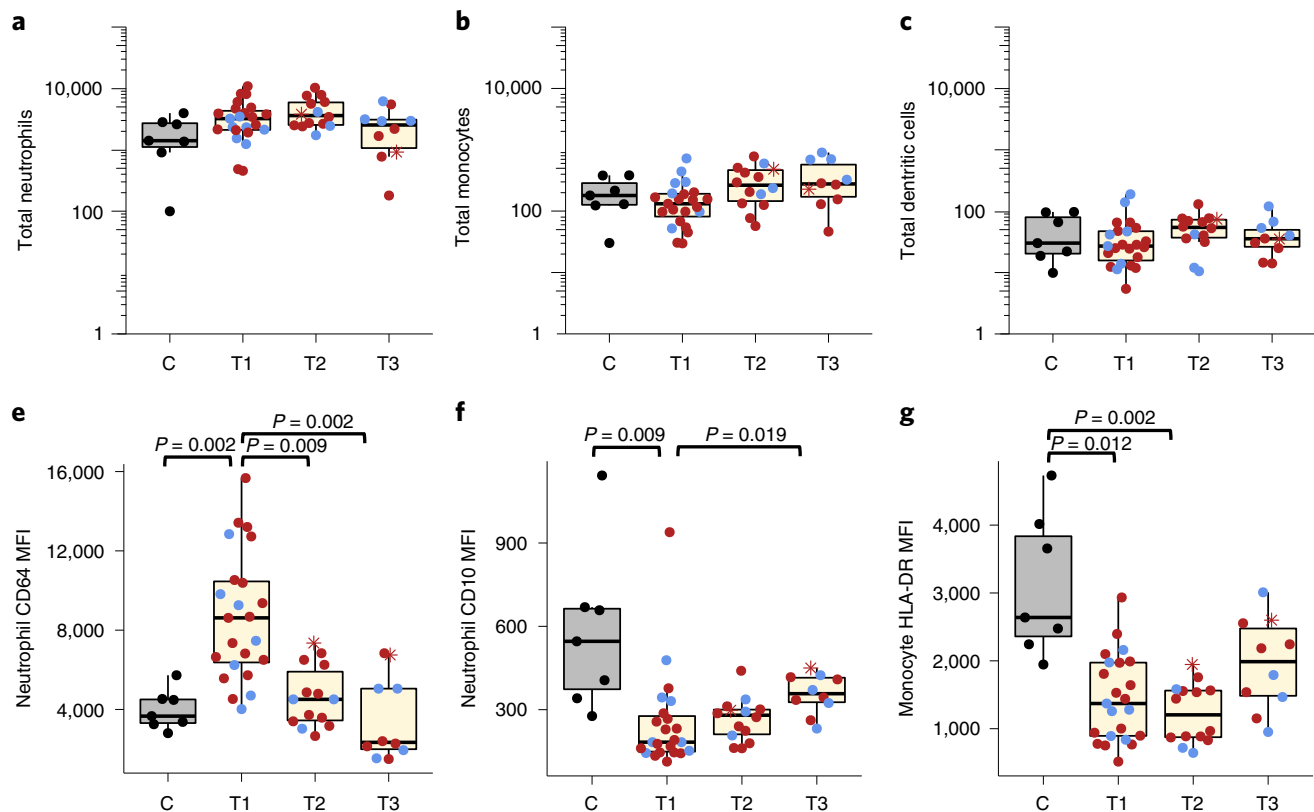

i

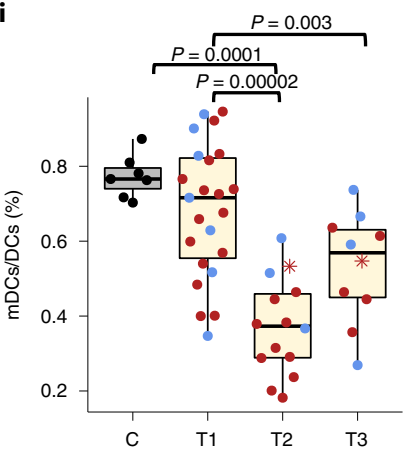

j

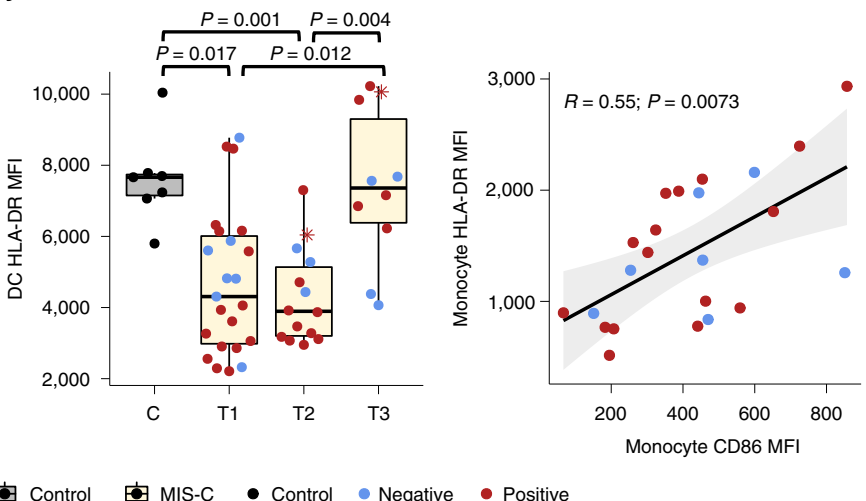

d

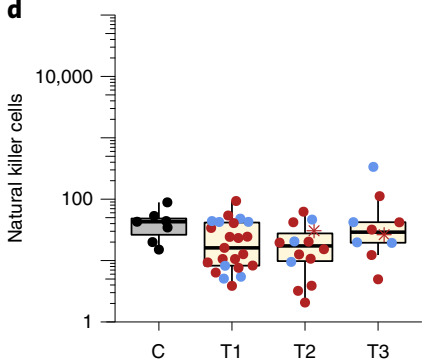

h

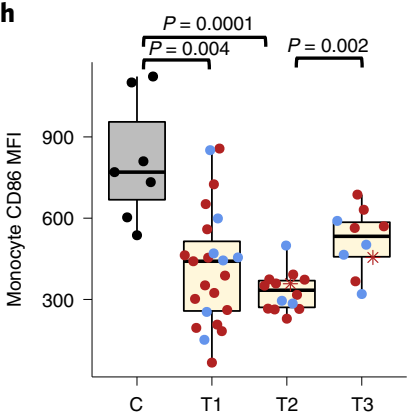

I

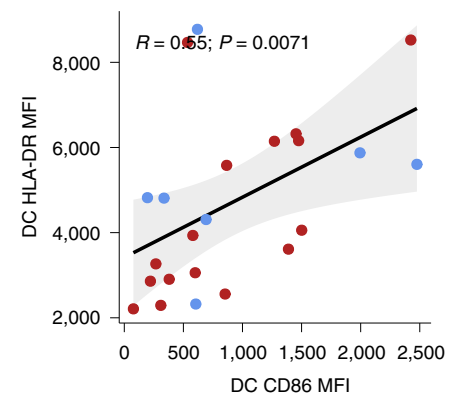

Fig. 2 | Innate immune cell alterations in patients with MIS-C at T1, T2 and T3 phases compared with age-matched healthy controls. a-d, Total neutrophil (a), monocyte (b), dendritic cell (c) and natural killer cell counts (d) per microliter of blood at each phase of disease. C, control. e-I, Neutrophil activation $(C D 64 \mathrm{MFI})(\mathbf{e})$, neutrophil CD10 expression (f), monocyte HLA-DR expression ( $\mathbf{g})$ and monocyte CD86 expression (h) at each phase of disease, myeloid dendritic cells ( $\mathrm{mDCs}$ ) as a percentage of total dendritic cells (DCs) (i), HLA-DR expression on dendritic cells (j), correlation between monocyte CD86 expression and monocyte HLA-DR expression (k), correlation between dendritic cell CD86 expression and dendritic cell HLA-DR expression (I). In $\mathbf{k}$ and $\mathbf{I}$, the regression line (solid) and $95 \% \mathrm{Cl}$ (gray shading) are shown. For the box and whisker plots, the bottom border represents the 25th percentile, the line bisecting the shaded region of the box represents the median, the upper border of the box represents the 75th percentile, the whiskers represent extreme values $(1.5 \times$ the 75 th (highest) and 25 th (lowest) percentile values) and markers beyond the extreme line are outliers. Dots are colored by serology result (red: positive; blue: negative), with the red asterisk indicating the patient who was positive by RT-PCR for SARS-CoV-2. Gray shading represents healthy controls and yellow shading represents patients with MIS-C. Further details are provided in Extended Data Fig. 4. A Spearman's rank test was used for the correlations. Significance testing between patients by phase of illness (T1 ( $n=23$ samples), T2 ( $n=14$ samples) and T3 ( $n=10$ samples)) was performed using two-sided Wilcoxon rank-sum tests with correction for multiple comparisons.

were the strongest features in later phases. We postulate that these represent either immunomodulatory treatment effects (reported in Fig. 1a and Table 1) or illness trajectory. Although previous MIS-C studies $^{2-13}$ included similar immunomodulatory treatments to those used in our cohort, they did not report corresponding longitudinal immunological assessments, to enable direct comparisons.

Immunologically, our MIS-C cohort appears distinct from Kawasaki disease as we did not observe neutrophilia and raised monocyte counts, which are features of Kawasaki disease. In Kawasaki disease, the CD4 and CD8 counts are higher ${ }^{37}$ than the $\mathrm{T}$ cell counts observed in our MIS-C cohort, and the proportions of HLA-DR-positive CD4 ${ }^{+} \mathrm{T}$ cells are lower in Kawasaki disease.
Activation of $\mathrm{CD} 4^{+} \mathrm{CCR} 7^{+} \mathrm{T}$ cells and $\gamma \delta \mathrm{T}$ cell subsets has not been reported in Kawasaki disease. Finally, Kawasaki disease is also characterized by activation of the IL- 1 pathways ${ }^{18,19}$, whereas altered IFN responses well described in COVID-19 may be more relevant in MIS-C ${ }^{1}$, such as the increased IFN- $\gamma$ levels observed in our cohort (Fig. 1d). Immunologically, although MIS-C shares features of adult COVID-19, such as cytokine excess and lymphopenia, there are differences, such as the higher neutrophil count and the increase in non-naive CD4 T cells with evidence of T cell exhaustion ${ }^{35,36}$. These differences indicate that MIS-C may be a distinct immunopathogenic illness, but concurrent immunophenotyping of Kawasaki disease and adult COVID-19 are needed for confirmation. 
a
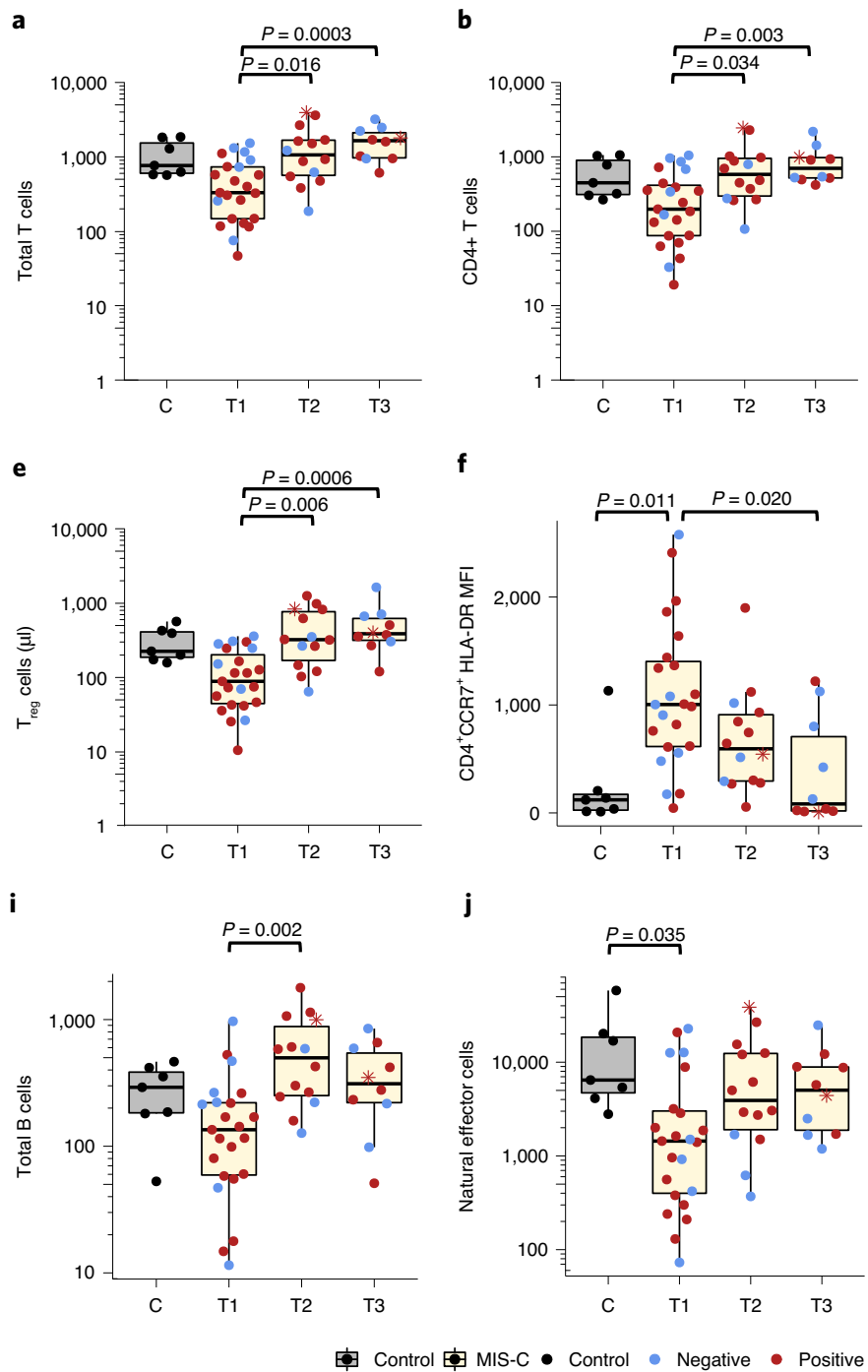

b

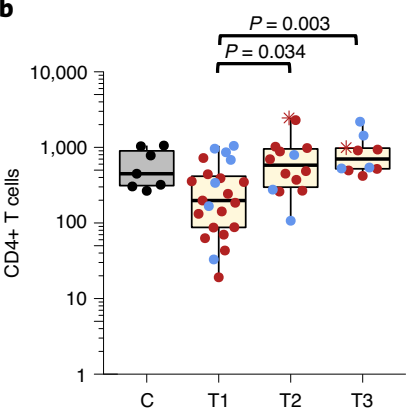

f

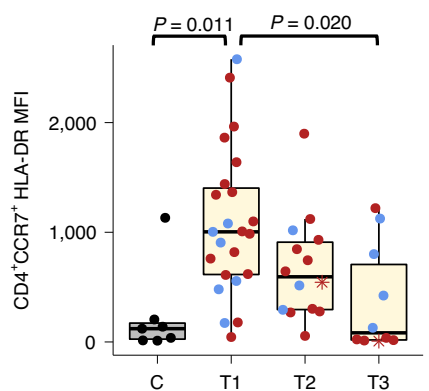

j

k

g

k
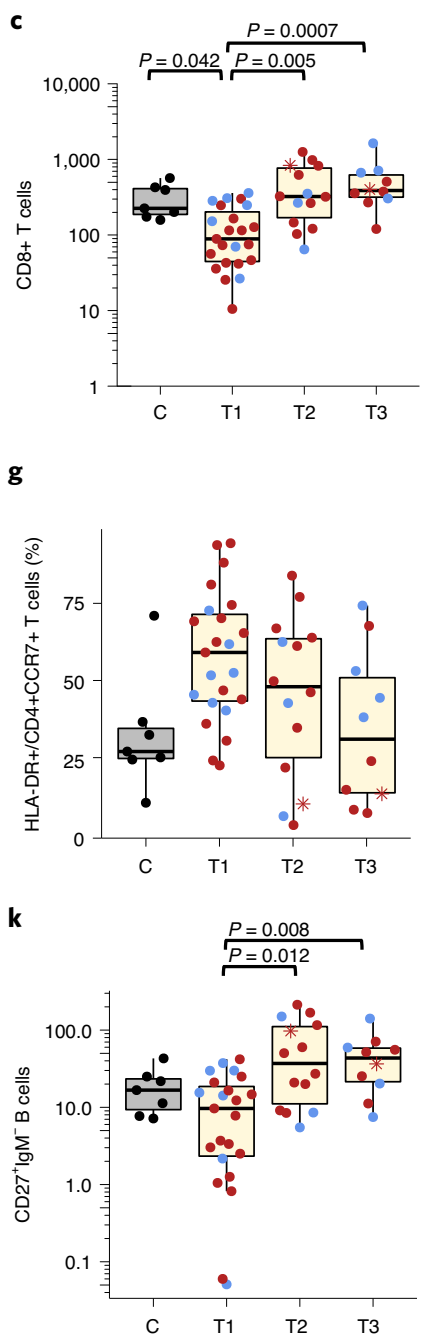

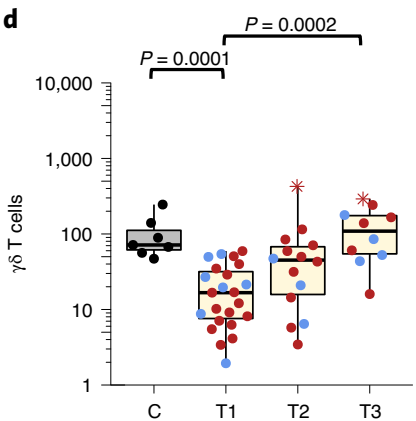

h

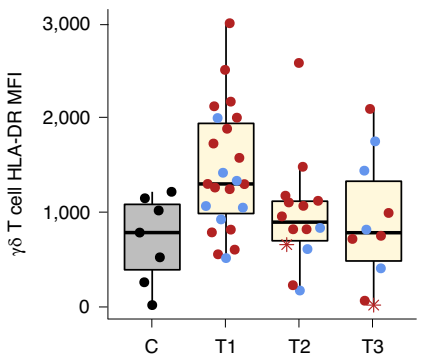

I

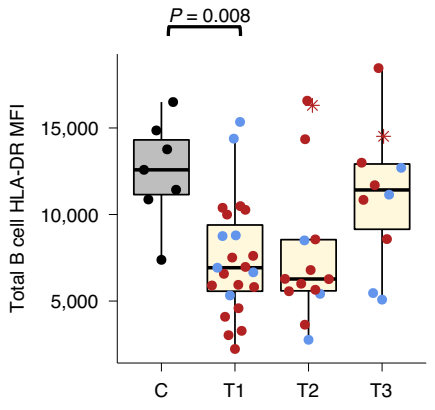

Fig. 3 | T and B cell alterations in patients with MIS-C at T1, T2 and T3 phases compared with age-matched healthy controls. a-e, Total T cells (a), CD4 ${ }^{+} \mathrm{T}$ cells (b), CD8 ${ }^{+} \mathrm{T}$ cells $(\mathbf{c}), \gamma \delta \mathrm{T}$ cells (d) and regulatory $\mathrm{T}$ cells ( $\mathrm{T}_{\text {reg }}$ cells) (e) per microliter of blood. f, CD4 ${ }^{+} \mathrm{CCR} 7^{+} \mathrm{T}$ cell HLA-DR $\mathrm{MFI}$ (activation marker). $\mathbf{g}$, Percentage of HLA-DR expressing CD4 ${ }^{+}$CCR7 ${ }^{+} \mathrm{T}$ cells. $\mathbf{h}, \gamma \delta \mathrm{T}$ cell HLA-DR MFI (activation marker). i-k, Total B cells (i) per microliter of blood, natural effector cells (j) per milliliter of blood and CD27+lgM- (naive) B cells (k) per microliter of blood. I, Total B cell HLA-DR expression $(\mathrm{MFI})$. For all box and whisker plots, the bottom border represents the 25th percentile, the line bisecting the shaded region of the box represents the median, the upper border of the box represents the 75th percentile, the whiskers represent extreme values (1.5x the 75th (highest) and 25th (lowest) percentile values) and markers beyond the extreme line are outliers. Dots are colored by serology result (red: positive; blue: negative), with the red asterisk indicating the patient who was positive by RT-PCR for SARS-CoV-2. Gray shading represents healthy controls and yellow shading represents patients with MIS-C. Further details are provided in Extended Data Fig. 5 . Significance testing between patients by phase of illness (T1 ( $n=23$ samples), T2 ( $n=14$ samples) and T3 ( $n=10$ samples)) was performed using two-sided Wilcoxon rank-sum tests with correction for multiple comparisons.

Our study was limited by the assessment of a small cohort from a tertiary pediatric center, the lack of testing for viral replication in the gastrointestinal $\operatorname{tract}^{38}$, the sole use of HLA-DR as a T cell activation marker and the lack of an evaluation of potential genetic susceptibilities $^{39}$. In addition, cell functionality and mechanisms of immune abnormalities were not measurable with cells preserved in leukocyte-stabilizing medium, which should be considered in future studies. The initiating trigger(s) for MIS-C are unclear. The two proposed immunopathogenic drivers of MIS-C (altered IFN responses and antibody-dependent enhancement of disease (ADE) ${ }^{1}$ ) may differ between patients. While altered IFN responses have indirect evidence from adult COVID-19 illness ${ }^{35,36}$, it is currently unclear whether ADE is a relevant mechanism in SARS-CoV-2-mediated immunopathogenesis in MIS-C. It is worth noting that in SARS and Middle East respiratory syndrome coronavirus infections, one of the mechanisms exacerbating lung injury is ADE-mediated viral entry into Fc receptor-expressing cells ${ }^{40,41}$. Further studies are required to confirm whether these mechanisms are relevant to MIS-C.

In conclusion, based on our cohort characteristics and the immune cell changes we observed, MIS-C is likely to be a distinct immunopathogenic illness associated with SARS-CoV-2, with more severe illness in seropositive children. The mechanisms underpinning these immune abnormalities are of priority for further research.

\section{Online content}

Any methods, additional references, Nature Research reporting summaries, source data, extended data, supplementary information, acknowledgements, peer review information; details of author contributions and competing interests; and statements of data and code availability are available at https://doi.org/10.1038/s41591-020-1054-6. 
Received: 1 July 2020; Accepted: 5 August 2020;

Published online: 18 August 2020

\section{References}

1. Rowley, A. H. Understanding SARS-CoV-2-related multisystem inflammatory syndrome in children. Nat. Rev. Immunol. 20, 453-454 (2020).

2. Verdoni, L. et al. An outbreak of severe Kawasaki-like disease at the Italian epicentre of the SARS-CoV-2 epidemic: an observational cohort study. Lancet 395, 1771-1778 (2020).

3. Riphagen, S., Gomez, X., Gonzalez-Martinez, C., Wilkinson, N. \& Theocharis, P. Hyperinflammatory shock in children during COVID-19 pandemic. Lancet 395, 1607-1608 (2020)

4. Cabrero-Hernandez, M. et al. Severe SARS-CoV-2 infection in children with suspected acute abdomen: a case series from a tertiary hospital in Spain. Pediatr. Infect. Dis. J. 39, e195-e198 (2020).

5. Belhadjer, Z. et al. Acute heart failure in multisystem inflammatory syndrome in children (MIS-C) in the context of global SARS-CoV-2 pandemic. Circulation 142, 429-436 (2020).

6. Chiotos, K. et al. Multisystem inflammatory syndrome in children during the coronavirus 2019 pandemic: a case series. J. Pediatr. Infect. Dis. Soc. 9, 393-398 (2020).

7. Whittaker, E. et al. Clinical characteristics of 58 children with a pediatric inflammatory multisystem syndrome temporally associated with SARS-CoV-2. JAMA 324, 259-269 (2020).

8. Ramcharan, T. et al. Paediatric inflammatory multisystem syndrome: temporally associated with SARS-CoV-2 (PIMS-TS): cardiac features, management and short-term outcomes at a UK tertiary paediatric hospital. Pediatr. Cardiol. 2020, 1-11 (2020).

9. Kaushik, S. et al. Multisystem inflammatory syndrome in children associated with severe acute respiratory syndrome coronavirus 2 infection: a multi-institutional study from New York City. J. Pediatr. https://doi. org/10.1016/j.jpeds.2020.06.045 (2020).

10. Capone, C. A. et al. Characteristics, cardiac involvement, and outcomes of multisystem inflammatory disease of childhood (MIS-C) associated with SARS-CoV-2 infection. J. Pediatr. https://doi.org/10.1016/j.jpeds.2020.06.044 (2020).

11. Toubiana, J. et al. Kawasaki-like multisystem inflammatory syndrome in children during the COVID-19 pandemic in Paris, France: prospective observational study. BMJ 369, m2094 (2020).

12. Feldstein, L. R. et al. Multisystem inflammatory syndrome in U.S. children and adolescents. N. Engl. J. Med. 383, 334-346 (2020).

13. Dufort, E. M. et al. Multisystem inflammatory syndrome in children in New York State. N. Engl. J. Med. 383, 347-358 (2020).

14. Sun, B. et al. Kinetics of SARS-CoV-2 specific IgM and IgG responses in COVID-19 patients. Emerg. Microbes Infect. 9, 940-948 (2020).

15. Long, Q. X. et al. Antibody responses to SARS-CoV-2 in patients with COVID-19. Nat. Med. 26, 845-848 (2020).

16. Guidance-Paediatric Multisystem Inflammatory Syndrome Temporally Associated with COVID-19 (PIMS) (Royal College of Paediatrics and Child Health, 2020); https://www.rcpch.ac.uk/resources/guidance-paediatricmultisystem-inflammatory-syndrome-temporally-associated-covid-19

17. Dallaire, F. \& Dahdah, N. New equations and a critical appraisal of coronary artery $Z$ scores in healthy children. J. Am. Soc. Echocardiogr. 24, 60-74 (2011).

18. Newburger, J. W., Takahashi, M. \& Burns, J. C. Kawasaki disease. J. Am. Coll. Cardiol. 67, 1738-1749 (2016).

19. Rowley, A. H. \& Shulman, S. T. The epidemiology and pathogenesis of Kawasaki disease. Front Pediatr. 6, 374 (2018).

20. Fernandez-Cooke, E. et al. Epidemiological and clinical features of Kawasaki disease in Spain over 5 years and risk factors for aneurysm development. (2011-2016): KAWA-RACE study group. PLoS ONE 14, e0215665 (2019).
21. Tacke, C. E. et al. Five years of Kawasaki disease in the Netherlands: a national surveillance study. Pediatr. Infect. Dis. J. 33, 793-797 (2014).

22. Long, Q. X. et al. Clinical and immunological assessment of asymptomatic SARS-CoV-2 infections. Nat. Med. 26, 1200-1204 (2020).

23. Wang, Y. \& Jonsson, F. Expression, role, and regulation of neutrophil fc $\gamma$ receptors. Front. Immunol. 10, 1958 (2019).

24. Marini, O. et al. Mature $\mathrm{CD} 10^{+}$and immature $\mathrm{CD} 10^{-}$neutrophils present in G-CSF-treated donors display opposite effects on T cells. Blood 129, 1343-1356 (2017).

25. Costa, S., Bevilacqua, D., Cassatella, M. A. \& Scapini, P. Recent advances on the crosstalk between neutrophils and B or T lymphocytes. Immunology 156, 23-32 (2019).

26. Zanoni, I. \& Granucci, F. Role of CD14 in host protection against infections and in metabolism regulation. Front. Cell Infect. Microbiol. 3, 32 (2013)

27. Krutmann, J. et al. Cross-linking Fc receptors on monocytes triggers IL-6 production. Role in anti-CD3-induced T cell activation. J. Immunol. 145, 1337-1342 (1990)

28. Tanaka, M. et al. Activation of Fc $\gamma$ RI on monocytes triggers differentiation into immature dendritic cells that induce autoreactive $\mathrm{T}$ cell responses. $J$. Immunol. 183, 2349-2355 (2009).

29. Freer, G. \& Matteucci, D. Influence of dendritic cells on viral pathogenicity. PLoS Pathog. 5, e1000384 (2009).

30. Maecker, H. T., McCoy, J. P. \& Nussenblatt, R. Standardizing immunophenotyping for the Human Immunology Project. Nat. Rev. Immunol. 12, 191-200 (2012).

31. Van den Broek, T., Borghans, J. A. M. \& van Wijk, F. The full spectrum of human naive T cells. Nat. Rev. Immunol. 18, 363-373 (2018).

32. Zheng, J., Liu, Y., Lau, Y. L. \& Tu, W. $\gamma \delta$-T cells: an unpolished sword in human anti-infection immunity. Cell. Mol. Immunol. 10, 50-57 (2013).

33. Vignali, D. A., Collison, L. W. \& Workman, C. J. How regulatory T cells work. Nat. Rev. Immunol. 8, 523-532 (2008).

34. Dono, M., Cerruti, G. \& Zupo, S. The CD5 ${ }^{+}$B-cell. Int. J. Biochem. Cell Biol. 36, 2105-2111 (2004)

35. Mathew, D. et al. Deep immune profiling of COVID-19 patients reveals distinct immunotypes with therapeutic implications. Science https://doi. org/10.1126/science.abc8511 (2020).

36. Laing, A. G. et al. A consensus COVID-19 immune signature combines immuno-protection with discrete sepsis-like traits associated with poor prognosis. Preprint at medRxiv https://doi.org/10.1101/2020.06.08.20125112 (2020).

37. Matsuguma, C. et al. Dynamics of immunocyte activation during intravenous immunoglobulin treatment in Kawasaki disease. Scand. J. Rheumatol. 48, 491-496 (2019)

38. Lamers, M. M. et al. SARS-CoV-2 productively infects human gut enterocytes. Science 369, 50-54 (2020).

39. Ellinghaus, D. et al. Genomewide association study of severe Covid-19 with respiratory failure. N. Engl. J. Med. https://doi.org/10.1056/NEJMoa2020283 (2020)

40. Wan, Y. et al. Molecular mechanism for antibody-dependent enhancement of coronavirus entry. J. Virol. 94, e02015-19 (2020).

41. Liu, L. et al. Anti-spike IgG causes severe acute lung injury by skewing macrophage responses during acute SARS-CoV infection. JCI Insight 4, e123158 (2019).

Publisher's note Springer Nature remains neutral with regard to jurisdictional claims in published maps and institutional affiliations.

(c) The Author(s), under exclusive licence to Springer Nature America, Inc. 2020 


\section{Methods}

Setting and approvals. Evelina London Children's Hospital is a major tertiary referral center for pediatric infectious diseases, cardiology and intensive care, and coordinates retrieval for 2 million children in South London and Southeast England-the region that had the highest number of COVID-19 confirmed cases in the United Kingdom during the pre-recruitment and recruitment window of our cohort (Extended Data Fig. 1). Our institution identified and reported one of the first case series of MIS- $\mathrm{C}^{3}$. The current cohort study was approved by the UK Health Research Authority (20/HRA/1714; for further details, please see https:// www.diamonds2020.eu/about/). Informed consent was obtained (from parents or guardians of children $<16$ years of age) by trained health professionals. The STROBE (Strengthening the Reporting of Observational Studies in Epidemiology) checklist compliance is provided in the Supplementary Information.

Case definitions. We recruited patients to this prospective cohort study between 27 April 2020 and 25 May 2020. The clinical features of 11 children included in this cohort have been previously been reported by the UK Paediatric Intensive Care Audit Network (PICANet) as part of a national cohort ${ }^{42}$. Children under the age of 18 years were potentially eligible for the study if febrile $\left(\geq 38.0^{\circ} \mathrm{C}\right)$. The Centres for Disease Control definition of MIS-C, the UK Royal College of Paediatrics and Child Health definition of PIMS-TS ${ }^{14}$ and the World Health Organization definitions are shown in Supplementary Table 1.

Clinical data. We collected data on age, sex, self-reported ethnicity, comorbidity, clinical features at presentation, organ dysfunction/support, immunomodulatory treatments given, worst sequential organ failure score (modified sequential organ failure assessment score variables were adjusted by age group; see Supplementary Table 2 for further details ${ }^{43}$ ) and the outcomes (hospital length of stay, admission to critical care (pediatric intensive care unit (PICU)), cardiac abnormalities (including left ventricular dysfunction, myocardial infarction and coronary artery dilation/aneurysms (defined as a Montreal $Z$ score $^{17}$ of $\geq 2$ on echocardiography or computed tomography)) and evidence of significant thrombi). Primary end-point pneumonia was defined per Cherian et al. ${ }^{44}$. We also collected leukocyte counts, hemoglobin, inflammation markers (CRP and ferritin), coagulation markers (D-dimer, fibrinogen and platelets) and markers of myocardial injury/dysfunction (troponin and NT-proBNP) at the three clinically relevant illness phases described earlier: acute (referred as T1); resolution (T2); and convalescent (T3).

Testing for other infections. We ruled out other acute infections using PCR to a panel of respiratory viral pathogens consisting of influenza A, influenza B, respiratory syncytial virus, enterorhinovirus, parainfluenza, adenovirus, human metapneumovirus, Bordetella species, Bordetella pertussis and Mycoplasma pneumoniae on nasopharyngeal or bronchoalveolar lavage culture of blood and other relevant tissues including urine, bronchoalveolar lavage specimens and cerebrospinal fluid, as indicated. Thus, only children meeting the criteria for MIS-C were included in this analysis.

Cytokine measurements in the MIS-C cohort at T1. Cytokines were all measured before the administration of intravenous immunoglobulin or other immunomodulatory treatment. Acute serum samples (pre-intravenous immunoglobulin administration) from 15 of the 25 children recruited had cytokines measured. The following cytokines were measured only at the T1 phase and only in the MIS-C cohort: IL-2 receptor agonist, IFN- $\gamma$, IL-10, IL-17, IL-1 $\beta$, IL-6, IL- 8 and tumor necrosis factor- $\alpha$. Serum was initially diluted 1:2 in distilled water. We used the Ella 600-100 (R\&D Systems) automated enzyme-linked immunosorbent (ELISA) platform within the Viapath laboratories at King's College Hospital, London, United Kingdom. This performs a sandwich ELISA using a microfluidics Simple Plex cartridge. This immunoassay works by routing the sample through a microfluidic channel that binds the protein of interest. The unbound analyte is removed by washing, before a detection antibody is added. Because each channel has three glass nano reactors coated with a capture antibody, a triplicate set of results are produced for each sample. Results were then generated using the manufacturer-calibrated standard curve.

SARS-CoV-2 infection status, serology and pseudoviral neutralization assays. We assessed the SARS-CoV-2 infection status of all patients by RT-PCR of respiratory samples.

Serology. We quantified IgG and IgM for spike protein, nucleocapsid and RBD using an in-house ${ }^{45}$ ELISA at T1, T2 and T3. We defined seropositivity as the IgG to both nucleocapsid and spike protein being at least fourfold above the assay background.

The nucleocapsid protein (comprising residues 48-365 and both ordered domains with the native linker, with an N-terminal uncleavable hexahistidine tag) was expressed in Escherichia coli using autoinducing media for $7 \mathrm{~h}$ at $37^{\circ} \mathrm{C}$. Protein was purified using immobilized metal affinity chromatography, size exclusion and heparin chromatography. The nucleocapsid protein was provided by L. James and J. Luptak at the Laboratory of Molecular Biology, Cambridge.
The spike protein (pre-fusion spike protein ectodomain residues 1-1,138, with a GGGG substitution at the furin cleavage site (amino acids 682-685), proline substitutions at amino acid positions 986 and 987 and an N-terminal T4 trimerization domain followed by a Strep-tag II) was expressed in HEK293F cells (Invitrogen). Briefly, a 11 culture (density 1.5 million cells per $\mathrm{ml}$ ) was transfected with $325 \mu \mathrm{g}$ DNA using PEI MAX ( $1 \mathrm{mg} \mathrm{ml}^{-1}$; Polysciences; $1: 3$ ratio) and cultured for $7 \mathrm{~d}$. Protein was purified using Strep-TactinXT Superflow high-capacity $50 \%$ suspension according to the manufacturer's protocol by gravity flow (IBA Lifesciences). The plasmid was provided by P. Brouwer, M. van Gils and R. Sanders at the University of Amsterdam.

The RBD was encoded by residues 319-541 and a carboxy-terminal hexahistidine tag for purification and expressed in HEK293F cells (Invitrogen). A 500 -ml culture (density of 1.5 million cells per $\mathrm{ml}$ ) was transfected with $1,000 \mu \mathrm{g}$ DNA using PEI MAX (1 $\mathrm{mg} \mathrm{ml}^{-1}$; Polysciences; $1: 3$ ratio) and cultured for $7 \mathrm{~d}$. Protein was purified using Ni-NTA agarose beads. The RBD plasmid was provided by F. Krammer at Mount Sinai University.

High-binding ELISA plates (Corning; 3690) were coated with SARS-CoV-2 antigen (nucleocapsid, spike protein or RBD) at $3 \mu \mathrm{g} \mathrm{ml}^{-1}(25 \mu \mathrm{l}$ per well) in phosphate-buffered saline (PBS), either overnight at $4^{\circ} \mathrm{C}$ or for $2 \mathrm{~h}$ at $37^{\circ} \mathrm{C}$. Wells were washed with PBS containing $0.05 \%$ Tween 20 (PBS-T) and blocked with $5 \%$ milk in PBS-T $(100 \mu \mathrm{l})$ for $1 \mathrm{~h}$ at room temperature. Wells were emptied and sera diluted at 1:50 in milk were added $(25 \mu \mathrm{l})$ and incubated for $2 \mathrm{~h}$ at room temperature. All sera used in this study were heat inactivated at $56^{\circ} \mathrm{C}$ for $30 \mathrm{~min}$ before use in the ELISA. The control reagents included CR3022 $\left(0.2 \mu \mathrm{g} \mathrm{ml}^{-1}\right.$; spike protein- and RBD-specific monoclonal antibody), CR3009 $\left(2 \mu \mathrm{g} \mathrm{ml}^{-1}\right.$; nucleocapsid-specific monoclonal antibody), negative control human plasma (1:25 dilution), positive control plasma (1:50) and blank wells (background control). Wells were washed five times with PBS-T. Secondary antibody was added $(25 \mu \mathrm{l})$ and incubated for $1 \mathrm{~h}$ at room temperature. IgM binding was detected using goat anti-human IgM-HRP (1:1,000; Sigma-Aldrich: A6907) and IgG binding was detected using goat anti-human Fc-AP (1:1,000; Jackson: 109-055-043-JIR). Wells were washed five times with PBS-T, and either alkaline phosphatase substrate (Sigma-Aldrich) was added $(25 \mu \mathrm{l})$ and read at $405 \mathrm{~nm}$ (goat anti-human Fc-AP) or one-step TMB substrate (Thermo Fisher Scientific) was added $(25 \mu \mathrm{l})$ and quenched with $\mathrm{H}_{2} \mathrm{SO}_{4}(25 \mu \mathrm{l}$ at $0.5 \mathrm{M}$ ) before reading at $450 \mathrm{~nm}$ (goat anti-human $\operatorname{IgM-HRP})^{45}$

Pseudoviral neutralization assays. $\mathrm{ID}_{50}$ measurements were determined using a virus neutralization assay with human immunodeficiency virus 1 (HIV-1) virus pseudotyped with SARS-CoV-2 full-length spike protein. Pseudotyped virus was expressed in HEK293T/17 cells. The day before transfection, $3.5 \times 10^{6}$ HEK293T/17 cells were seeded in a $10-\mathrm{cm}$ dish in complete Dulbecco's modified Eagle's medium (DMEM-C; $10 \%$ (vol/vol) fetal bovine serum, $100 \mathrm{IU} \mathrm{ml}^{-1}$ penicillin and $100 \mu \mathrm{g} \mathrm{ml}^{-1}$ streptomycin). Cells were transfected with HIV-luciferase plasmid (1,500 ng), HIV $8.91 \mathrm{gag} / \mathrm{pol}$ plasmid (1,000 ng) and SARS-CoV-2 full-length spike protein plasmid ( $900 \mathrm{ng}$; provided by N. Temperton) ${ }^{46}$ using $35 \mu \mathrm{g}$ PEI MAX (at $1 \mathrm{mg} \mathrm{ml}^{-1}$; Polysciences). DMEM-C media was replaced $18 \mathrm{~h}$ post-transfection. Virus particles were harvested after $48 \mathrm{~h}$, filtered through a $0.45-\mu \mathrm{m}$ filter and stored at $-80^{\circ} \mathrm{C}$. For the neutralization assays, sera (heat inactivated at $56^{\circ} \mathrm{C}$ for $30 \mathrm{~min}$ ) was serially diluted (DMEM-C) in 96-well plates and incubated with pseudovirus for $1 \mathrm{~h}$ at $37^{\circ} \mathrm{C}$. HeLa-ACE2 cells (HeLa cells stably expressing the angiotensin-converting enzyme 2 receptor provided by J. Voss at The Scripps Research Institute) were added. The infection level was assessed after $72 \mathrm{~h}$ in lysed cells with the Bright-Glo luciferase kit (Promega) using a VICTOR X3 multilabel reader (PerkinElmer) ${ }^{47}$

Leukocyte phenotyping. Healthy control for flow cytometry. We recruited healthy children $(n=7)$ of similar age, without any history of recent infection or immunological comorbidity, and collected blood samples as healthy controls for comparison with the MIS-C cohort, during the study period (Supplementary Table 3).

Blood sampling. Blood sampling consisted of $0.5 \mathrm{ml}$ whole blood in a leukocyte-stabilizing medium (Cytodelics AB; http://www.cytodelics.com/ uploads/1/2/3/5/123585057/whole_blood_processing_kit_protocol_ver106. pdf), obtained with routine clinical samples, at the three clinically relevant illness phases described earlier: acute (referred as T1); resolution (T2); and convalescent (T3). Whole-blood sample staining was performed. Briefly, frozen blood samples were thawed for $1 \mathrm{~min}$ in a $37^{\circ} \mathrm{C}$ water bath by gently swirling. Then, $200 \mu \mathrm{l} \mathrm{blood}$ was added to a 96-well V-bottom plate and spun at 2,000 r.p.m. for $2 \mathrm{~min}$. Cells were resuspended in $100 \mu \mathrm{l}$ staining mix and incubated at room temperature for $20 \mathrm{~min}$. All fluorochromes, clones and concentrations used for whole-blood surface staining for flow cytometry are described. Flow Cytometry Standard files, acquired using BD FACSDiva, were analyzed using FlowJo (version 10.6.2). Gating strategies for all panels used the Human Immunology Project ${ }^{30}$ approach. Event counts and MFIs were calculated using FlowJo for relevant markers on specific populations. Absolute cell counts were calculated using BioLegend Precision Count Beads. 
Whole-blood stabilization. Whole blood was collected on the PICU. Each $0.5-\mathrm{ml}$ sample of blood was immediately mixed with $0.5 \mathrm{ml}$ Cytodelics Stabilizer buffer (1:1 ratio), incubated at room temperature for $10 \mathrm{~min}$ and stored at $-80^{\circ} \mathrm{C}$, as described by the manufacturer (Cytodelics $\mathrm{AB})^{23}$.

Immune cell panels. We designed three different flow cytometry panels for standardized immunophenotyping of innate, T and B lymphocyte subsets, per the Human Immunology Project ${ }^{30}$. All fluorochromes were purchased from BD Biosciences-EU.

The innate immune cell panel consisted of the following targets (fluorochrome; clone; catalog number; antibody volume in $\mu \mathrm{l}$ per $100 \mu \mathrm{l}$ ): CD15 (BV786; HI98; 563838; 1.0); CD14 (BV421; M5E2; 565283; 1.0); CD56 (BB515; B159; 564489; 2.0); CD86 (BUV737; 2331; 612784; 1.0); CD68 (PE-CF594; Y1/82A; 564944; 1.0 ); HLA-DR (BV510; G46-6; 563083; 1.0); CD11c (BV650; B-ly6; 563404; 1.0); CD123 (PerCP-Cy5.5; 7G3; 560904; 1.0); CD16 (Pe-Cy7; 3G8; 560918; 1.0); CD64 (AF700; 10.1; 561188; 2.0); CD161 (PE; DX12; 556081; 1.0); CD10 (BUV395; HI10a; 563871; 3.0); CD19 (APC-Cy7; SJ25C1; 557791; 1.0); and CD3 (APC-Cy7; SK7; $560176 ; 1.0)$.

The $\mathrm{T}$ cell panel consisted of the following targets (fluorochrome; clone; catalog number; antibody volume in $\mu \mathrm{l}$ per $100 \mu \mathrm{l}$ ): CD3 (BUV395; SK7; 564001; 2.0); CD4 (BV786; SK3; 563877; 2.0); CD8 (BV605; SK1; 564116; 2.0); gamma-delta TCR (PE-Cy7; 11F2; 655410; 5.0); CCR7 (CD197) (BV421; 2-L1-A; 566743; 2.0); CCR4 (CD194) (PE-CF594; 1G1; 565391; 2.0); CCR6 (CD196) (BB515; 11Ag; 564479; 2.0); CD45RO (PE;UCHL1; 555493; 5.0); CD45RA (APC; HI100; 550855; 5.0); CXCR3 (CD183) (BB700; 1C6; 566532; 2.0); CD25 (BV510; M-A251; 563352; 2.0); CD25 (BV510; 2A3; 740198; 2.0); HLA-DR (APC-R700; G46-6; 565127; 2.0); and CD127 (BUV737; HIL-7R-M21; 612794; 2.0).

The B cell panel consisted of the following targets (fluorochrome; clone; catalog number; antibody volume in $\mu \mathrm{l}$ per $100 \mu \mathrm{l}$ ): CD19 (BV711; SJ25C1; 563038; 2.0); CD27 (BV786; L128; 563327; 2.0); CD43 (BV421; 1G10; 562916; 2.0); CD24 (BUV395; ML5; 563818; 2.0); IgG (APC; G18-145; 550931; 5.0); IgD (BUV737; IA6-2; 612798; 2.00); IgM (BB515; G20-127; 564622; 2.0); CD38 (PE; HIT-2; 555460; 10.0); CD5 (PE-Cy7; L17F12; 348810; 2.0); CD25 (APC-R700; 2A3; 565106; 2.0); and HLA-DR (BV510; G46-6; 563083; 2.0).

Flow cytometry and acquisition. Whole-blood sample staining was done using a Cytodelics processing kit (Cytodelics AB). Frozen blood samples were thawed for $1 \mathrm{~min}$ in a $37^{\circ} \mathrm{C}$ water bath by gently swirling. Then, $200 \mu \mathrm{l}$ of blood was added for each panel (innate, $\mathrm{T}$ cell and B cell) to a 96-well V-bottom plate and centrifuged at 2,000 r.p.m. for $2 \mathrm{~min}$ (centrifugation settings remained the same throughout). Cells were resuspended in $100 \mu \mathrm{l}$ of the respective antibody cocktail and incubated at room temperature for $20 \mathrm{~min}$. All flow cytometry antibodies and concentrations used for whole-blood surface staining can be found in Supplementary Table 4. PBS ( $100 \mu \mathrm{l}$; Gibco) was added to each well and the plate was centrifuged. Supernatant was removed and $200 \mu$ l fixative buffer (Cytodelics; 1:1 dilution of Fix Concentrate in Fix Diluent) was added. The plate was incubated at room temperature for $15 \mathrm{~min}$ and centrifuged and the supernatant was removed. Cells were resuspended in $200 \mu \mathrm{l}$ lysis buffer (Cytodelics; 1:4 dilution of lysis buffer in distilled $\mathrm{H}_{2} \mathrm{O}$ ) and incubated at room temperature for $15 \mathrm{~min}$. The plate was centrifuged and the supernatant was removed. Cells were resuspended in $200 \mu \mathrm{l}$ wash buffer (Cytodelics; 1:5 dilution of wash buffer concentrated in distilled $\mathrm{H}_{2} \mathrm{O}$ ) and centrifuged. The supernatant was removed and the cells were resuspended in $175 \mu \mathrm{l}$ sterile PBS. Before acquisition, $25 \mu \mathrm{l}$ Precision Count Beads (BioLegend) were added to each sample. All samples were analyzed on a five-laser BD Fortessa flow cytometer equipped with a BD High Throughput Sampler with a flow rate of $1 \mu \mathrm{l} \mathrm{s}^{-1}$. The flow cytometer was set up with application settings using cytometer set up and tracking beads (Becton Dickson).

Flow cytometry data analysis. Flow Cytometry Standard files were acquired using BD FACSDiva and analyzed using FlowJo (10.6.2; Treestar). Gating strategies for all panels are outlined in Supplementary Fig. 1 for innate immune cells, Supplementary Fig. 2 for T cells and Supplementary Fig. 3 for B cells. Event counts and median fluorescence intensities were calculated using FlowJo for relevant markers on specific populations. Absolute cell counts using BioLegend Precision Count Beads were calculated using the following equation:

$\begin{aligned} & \text { Absolute cell count }(\text { cells per } \mu \mathrm{l})= \\ & (\text { cell count } \times \text { Precision Count Beads volume })\end{aligned}$
(Precision Count Beads count $\times$ whole blood volume $)$
$\times$ bead concentration

Statistical analysis. Continuous data were summarized as medians and IQRs and categorical data are presented as frequencies and percentages. We did not perform a priori sample size calculations. Due to the limited sample size, the statistical significance reported should only be interpreted as indicative of the direction of change in biological signals. To compare differences between healthy controls and MIS-C illness phases (T1, T2 and T3), we represent data using box and whisker plots showing all of the data points using the Tidyverse package ${ }^{48}$, and used the Wilcoxon signed-rank test with Bonferroni correction for multiple comparisons. The strength and direction of association between variables was assessed using Spearman's correlation. We performed a principal component analyses (colored by illness phases and by receipt of immunomodulation) implemented using the Factoextra R package ${ }^{49}$. We generated a heatmap of all of the reported immunophenotyping parameters using the pheatmap $\mathrm{R}$ package ${ }^{50}$. All analyses were performed using the $\mathrm{R}$ studio interface ${ }^{51}$ and $\mathrm{R}^{52}$.

Reporting Summary. Further information on research design is available in the Nature Research Reporting Summary linked to this article.

\section{Data availability}

We have provided all of the deidentified raw data used in this report in the Supplementary Data, representing data in the figures and tables reported in the manuscript.

\section{References}

42. Davies, P. et al. Intensive care admissions of children with paediatric inflammatory multisystem syndrome temporally associated with SARS-CoV-2 (PIMS-TS) in the UK: a multicentre observational study. Lancet Child Adolesc. Health https://doi.org/10.1016/S2352-4642(20)30215-7 (2020).

43. Matics, T. J. \& Sanchez-Pinto, L. N. Adaptation and validation of a pediatric sequential organ failure assessment score and evaluation of the Sepsis-3 definitions in critically Ill children. JAMA Pediatr. 171, e172352 (2017).

44. Cherian, T. et al. Standardized interpretation of paediatric chest radiographs for the diagnosis of pneumonia in epidemiological studies. Bull. World Health Organ. 83, 353-359 (2005).

45. Pickering, S. et al. Comparative assessment of multiple COVID-19 serological technologies supports continued evaluation of point-of-care lateral flow assays in hospital and community healthcare settings. Preprint at medRxiv https://doi.org/10.1101/2020.06.02.20120345 (2020).

46. Grehan, K., Ferrara, F. \& Temperton, N. An optimised method for the production of MERS-CoV spike expressing viral pseudotypes. MethodsX 2 , 379-384 (2015)

47. Seow, J. et al. Longitudinal evaluation and decline of antibody responses in SARS-CoV-2 infection. Preprint at medRxiv https://doi. org/10.1101/2020.07.09.20148429 (2020).

48. Wickham, H. et al. Welcome to the Tidyverse. J. Open Source Softw. 4, 1686 (2019).

49. Kassambara, K. \& Mundt, F. factoextra: Extract and visualize the results of multivariate data analyses. R package version 1.0.7 https://cran.r-project.org/ web/packages/factoextra/index.html (2020)

50. Kolde, R. pheatmap: Pretty heatmaps. R package version 1.0.12 https:// cran.r-project.org/web/packages/pheatmap/index.html (2019).

51. RStudio Team RStudio: Integrated Development for $R$ (RStudio, 2020).

52. R Development Core Team R: A Language and Environment for Statistical Computing (R Foundation for Statistical Computing, 2020); http:// www.R-project.org/

\section{Acknowledgements}

We thank the parents and children who agreed to take part in this work. We thank J. Irons and the Evelina DIAMONDS Study team, the Evelina PIMS-TS Working Group and the Evelina Paediatric Research Team. We thank the flow cytometry core at the King's College London (KCL) NIHR Biomedical Research Centre, and for logistical support we thank M. Brown, R. Ellis, S. Cochrane and C. Trouillet. We thank F. Krammer (Icahn School of Medicine at Mount Sinai) for provision of the RBD expression plasmid, P. Brouwer, M. van Gils and R. Sanders (University of Amsterdam) for the spike protein expression plasmid, and L. James, J. Luptak and L. Kiss (LMB, Cambridge) for the provision of purified nucleocapsid protein. The development of SARS-CoV-2 reagents (RBD) was partially supported by the NIAID Centers of Excellence for Influenza Research and Surveillance (CEIRS) contract HHSN272201400008C. The study was supported by the King's Together COVID-19 seed funds (M.J.C., M.S.H., K.J.D., S.N and M.H.M.). M.J.C. is supported by a National Institute for Health Research (NIHR) Academic Clinical Lectureship. M.F. is supported by a National Institute of Academic Anesthesia BJA/RCoA fellowship (WKRO-2018-0047). A.J. is supported by PhD funds from the Critical Care Department at Guy's and St Thomas' Hospital. S.A. was supported by an MRC-KCL Doctoral Training Partnership in Biomedical Sciences Industrial Collaborative Award in Science and Engineering (iCASE) in partnership with Orchard Therapeutics (MR/R015643/1). C.G. is supported by the MRC-KCL Doctoral Training Partnership in Biomedical Sciences (MR/N013700/1). M.S.-H. is supported by the NIHR Clinician Scientist Award (CS-2016-16-011). The views expressed in this publication are those of the authors and not necessarily those of the NHS, NIHR or Department of Health and Social Care. This research was funded/supported by the NIHR Biomedical Research Centre based at Guy's and St Thomas' NHS Foundation Trust and KCL and/ or the NIHR Clinical Research Facility. The funding sources did not have any role in the design, conduct or interpretation of the study results.

\section{Author contributions}

M.J.C., P.W., J.K., M.F., A.J., S.M.T. and M.S.-H. had full access to all of the data in the study and take responsibility for the integrity of the data. K.J.D., S.A., J.S. and C.G. performed the ELISA and viral neutralization assays. M.F., A.J. and E.T. performed 
all of the flow cytometry optimization and analyses. M.J.C., M.F. and M.S.-H. take responsibility for the accuracy of the data analyses. M.J.C. and M.S.-H. conceived of and designed the study. M.J.C., M.F. and M.S.-H. performed the statistical analysis. M.J.C., M.F., A.J. and M.S.-H. drafted the manuscript. All authors contributed to acquisition, analysis and interpretation of the data and critical revision of the manuscript for important intellectual content. M.J.C., S.M.T. and M.S.-H. obtained the funding. S.M.T. and M.S.-H. provided administrative, technical or material support. M.S.-H. supervised the study.

\section{Competing interests}

The authors declare no competing interests.

\section{Additional information}

Extended data Extended data is available for this paper at https://doi.org/10.1038/ s41591-020-1054-6.

Supplementary information Supplementary information is available for this paper at https://doi.org/10.1038/s41591-020-1054-6.

Correspondence and requests for materials should be addressed to S.M.T. or M.S.-H.

Peer review information Saheli Sadanand was the primary editor on this article and managed its editorial process and peer review in collaboration with the rest of the editorial team.

Reprints and permissions information is available at www.nature.com/reprints. 


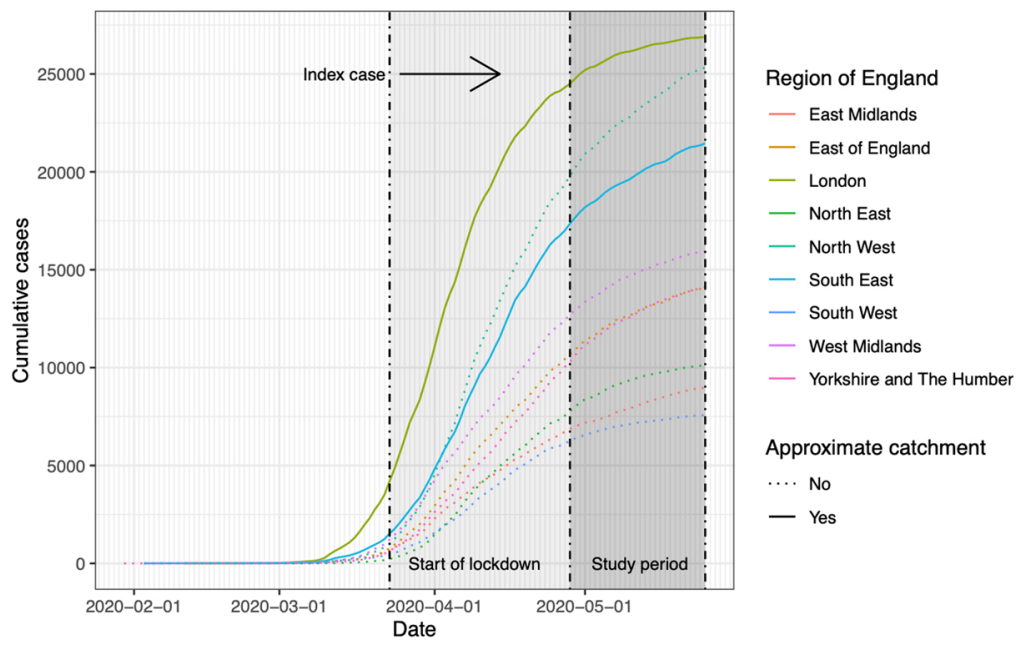

c

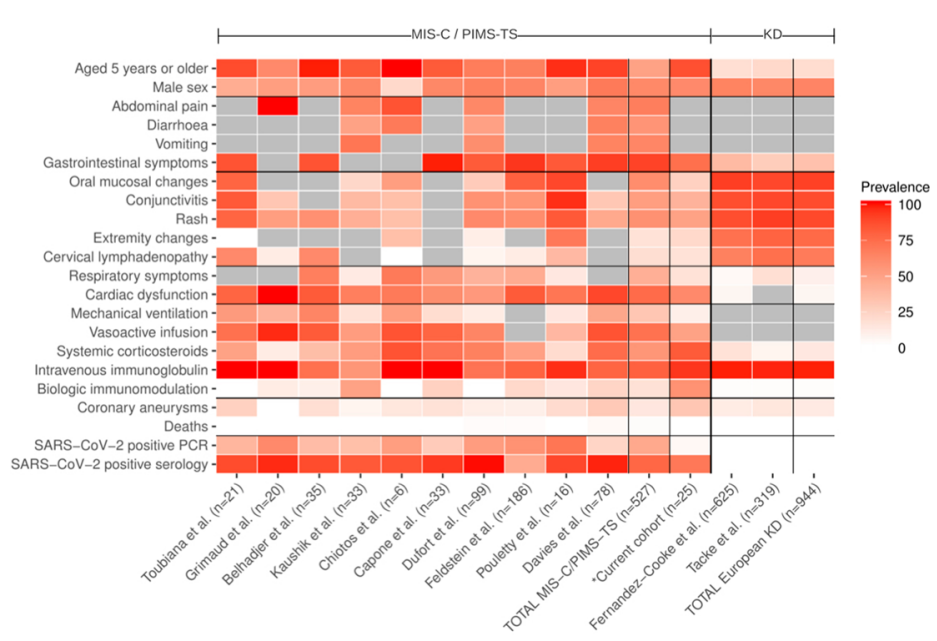

b

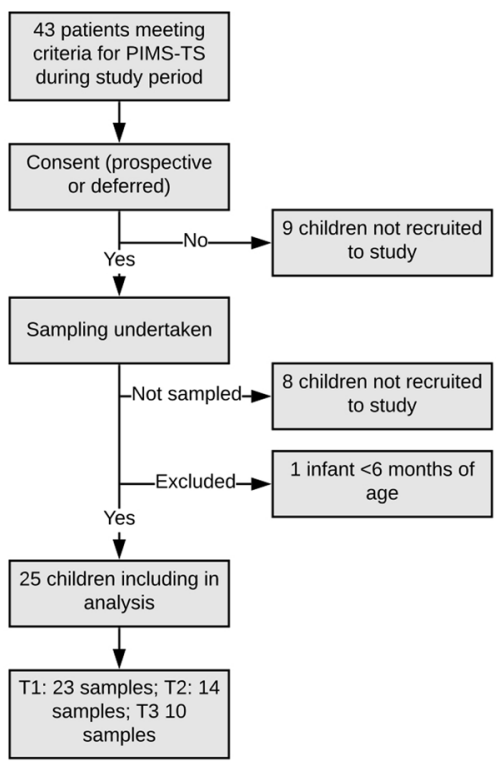

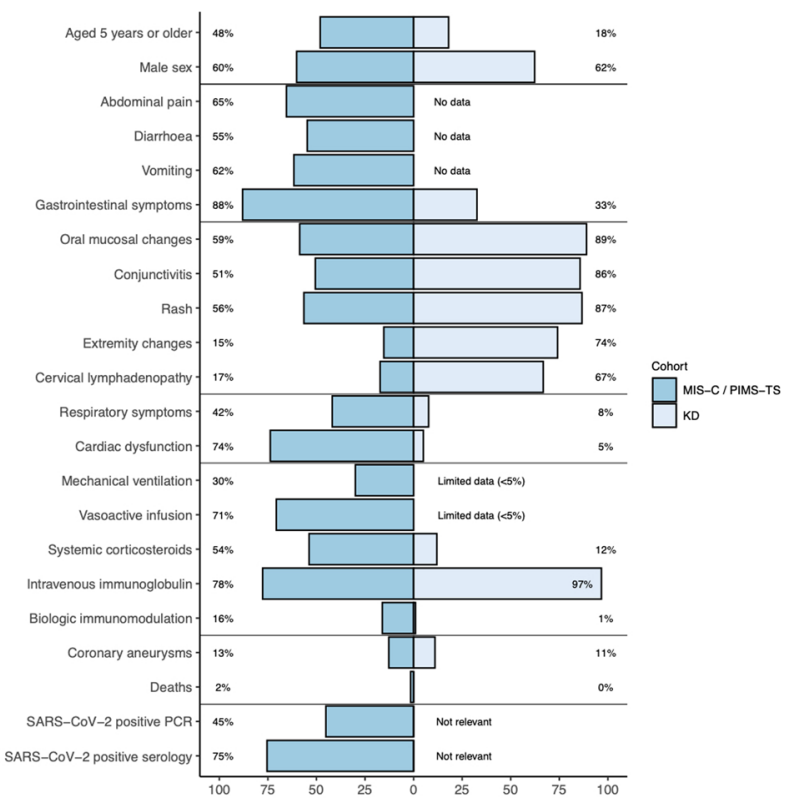

Extended Data Fig. 1 | Context of the study cohort. a, Epidemiology of SARS-CoV-2 infection in England prior to, and during the study period by region. Evelina London Children's Hospital is in central London and provides tertiary immunology, infectious diseases, cardiology and intensive care to children in south-east England in collaboration with other tertiary centers (referred to 'approximate catchment'). Data from the UK Government (https://coronavirus. data.gov.uk/) accessed on $22^{\text {nd }}$ June 2020. 'Index case' denotes the first case of PIMS-TS identified at Evelina London Children's Hospital. b, Flow chart of recruitment to the study cohort. c, Heat map of prevalence of clinical features in MIS-C (PIMS-TS in UK terminology, and Kawasaki-like disease in some continental European cohorts (Supplementary Table 4)) and two recent European Kawasaki disease (KD) cohorts. d, Clinical features of MIS-C / PIMS-TS in comparison with two recent European KD cohorts. 
a

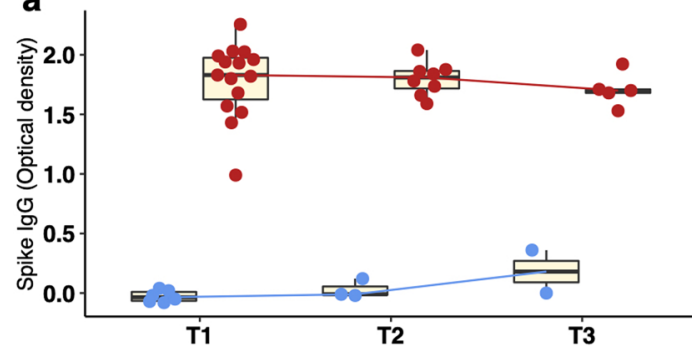

C

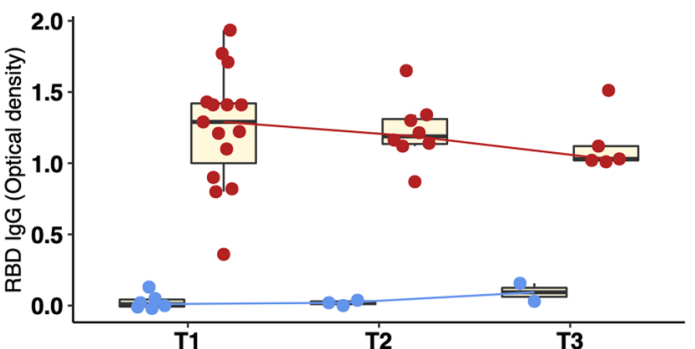

b

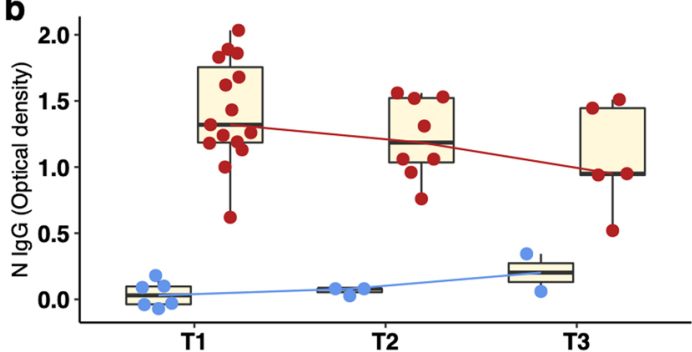

d

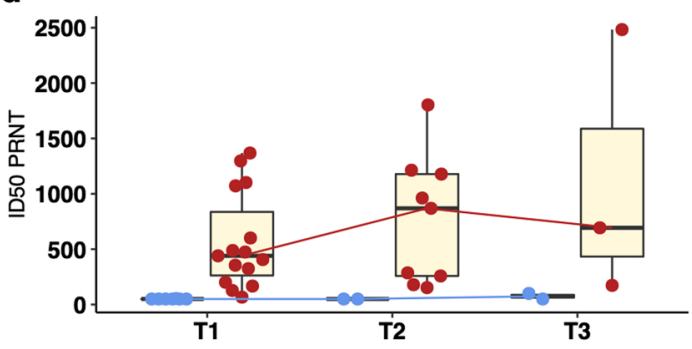

Serology 官 Negative 审 Positive

Extended Data Fig. 2 | Time course analysis of Spike, N, RBD and Pseudoviral neutralization assay (plaque reduction neutralization test) by serology to SARS-CoV-2 in patients with MIS-C at T1, T2 and T3 phases. a, IgG to S; b, IgG to N; c, IgG to RBD; children with MIS-C at T1 (acute), T2 (resolution) and T3 (convalescence). Seronegative and seropositive children are plotted as blue and red respectively; a single PCR positive child was plotted as an open red circle with cross. Antibody concentrations were measured by ELISA and reported as optical density (absorbance). d, Pseudoviral neutralization assay at T1, T2 and T3. Abbreviations: ID50, 50\% inhibitory dose. In figures, box and whisker plots, bottom border represents 25th percentile; line bisecting the shaded region of each box represents median; upper border of the box, 75th percentile. The whiskers represent extreme 1.5 times the 75th (highest) and 25th (lowest) values and markers beyond the extreme line are outliers. Dots are colored by serology result; positive (red) and negative (blue). No statistical testing was done between patients by phase of illness (T1 ( $n=21$ samples), T2 ( $n=11$ samples), T3 ( $n=7$ samples). 


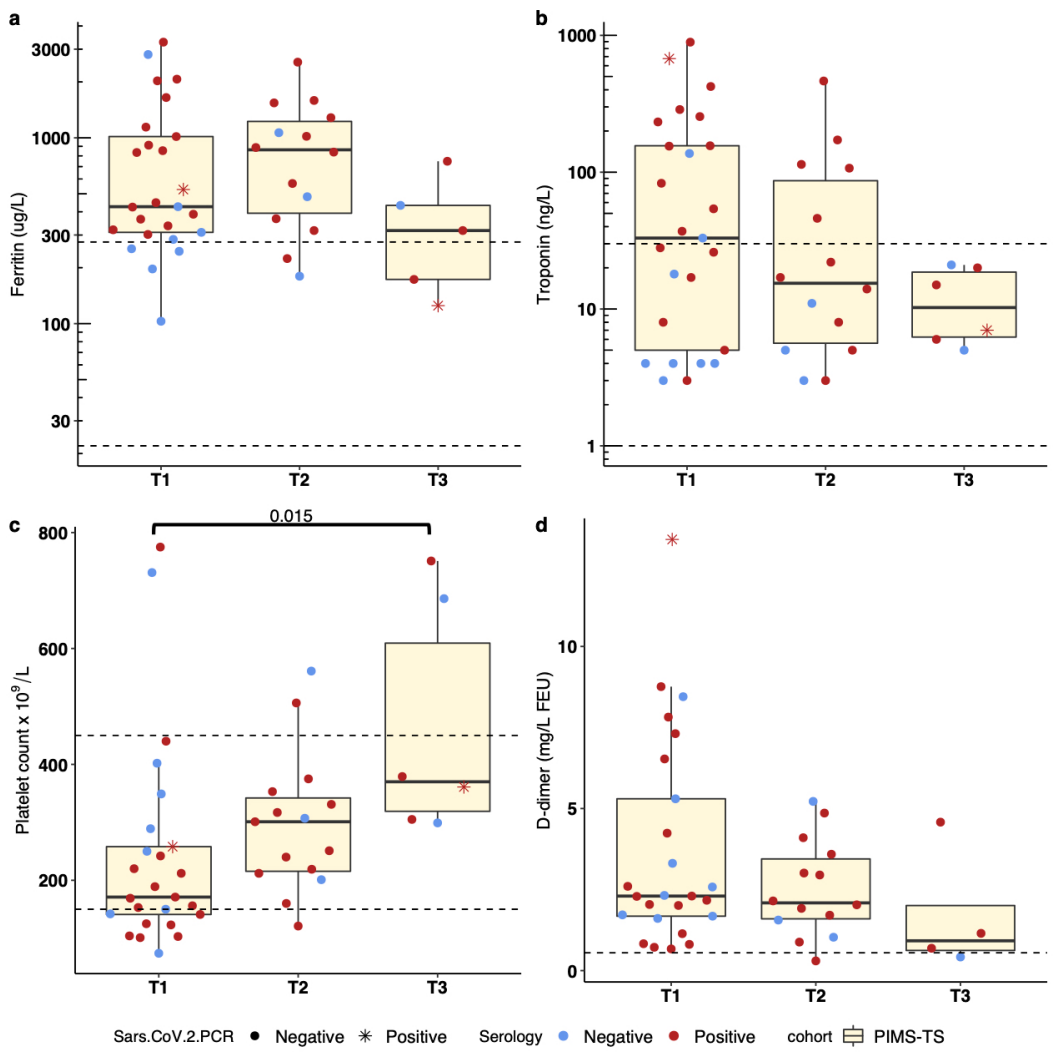

Extended Data Fig. 3 | Clinical biomarkers in patients with MIS-C at T1, T2 and T3 phases. a, Serum ferritin concentrations. decreased from acute and resolution illness phases ( $\mathrm{T} 1$ and $\mathrm{T} 2$ ) to convalescence ( $\mathrm{T} 3$; normal range shown as dashed lines, $22-275 \mu \mathrm{g} / \mathrm{L}$ ). $\mathbf{b}$, Serum troponin T concentrations were raised in most children in the acute illness phase ( $\mathrm{T} 1)$ and normalized by convalescence ( $\mathrm{T} 3$; threshold for acute myocardial infarction in adults shown as dashed line, $30 \mathrm{ng} / \mathrm{L}$ ). c, Blood platelet count increased significantly across time points from a relative thrombocytopenia in the acute phase (T1) to thrombocytosis by convalescence (T3; normal range shown as dashed lines, $150-450 \times 10^{9} / \mathrm{L}$ ). d, Serum D-dimer concentrations were elevated in the acute and resolution illness phases (T1 and T2) and decreased in convalescence (T3; upper threshold of normal shown as dashed line, $0.55 \mathrm{mg} / \mathrm{L}$ FEU). In figures, box and whisker plots, bottom border represents 25th percentile; line bisecting the shaded region of each box represents median; upper border of the box, 75th percentile. The whiskers represent extreme 1.5 times the 75th (highest) and 25th (lowest) values and markers beyond the extreme line are outliers. Dots are coloured by serology result; positive (red) and negative (blue), with the red asterisk indicating the patient positive by PCR for SARS-CoV-2. Significance testing between patients by phase of illness (T1 ( $n=25$ samples), T2 ( $n=15$ samples), T3 ( $n=6$ samples) was using two-sided Wilcoxon rank sum tests, with correction for multiple comparisons. Note $\log _{10}$ scales for $\mathbf{a}$ and $\mathbf{b}$. Abbreviations: FEU, fibrinogen equivalent units. 

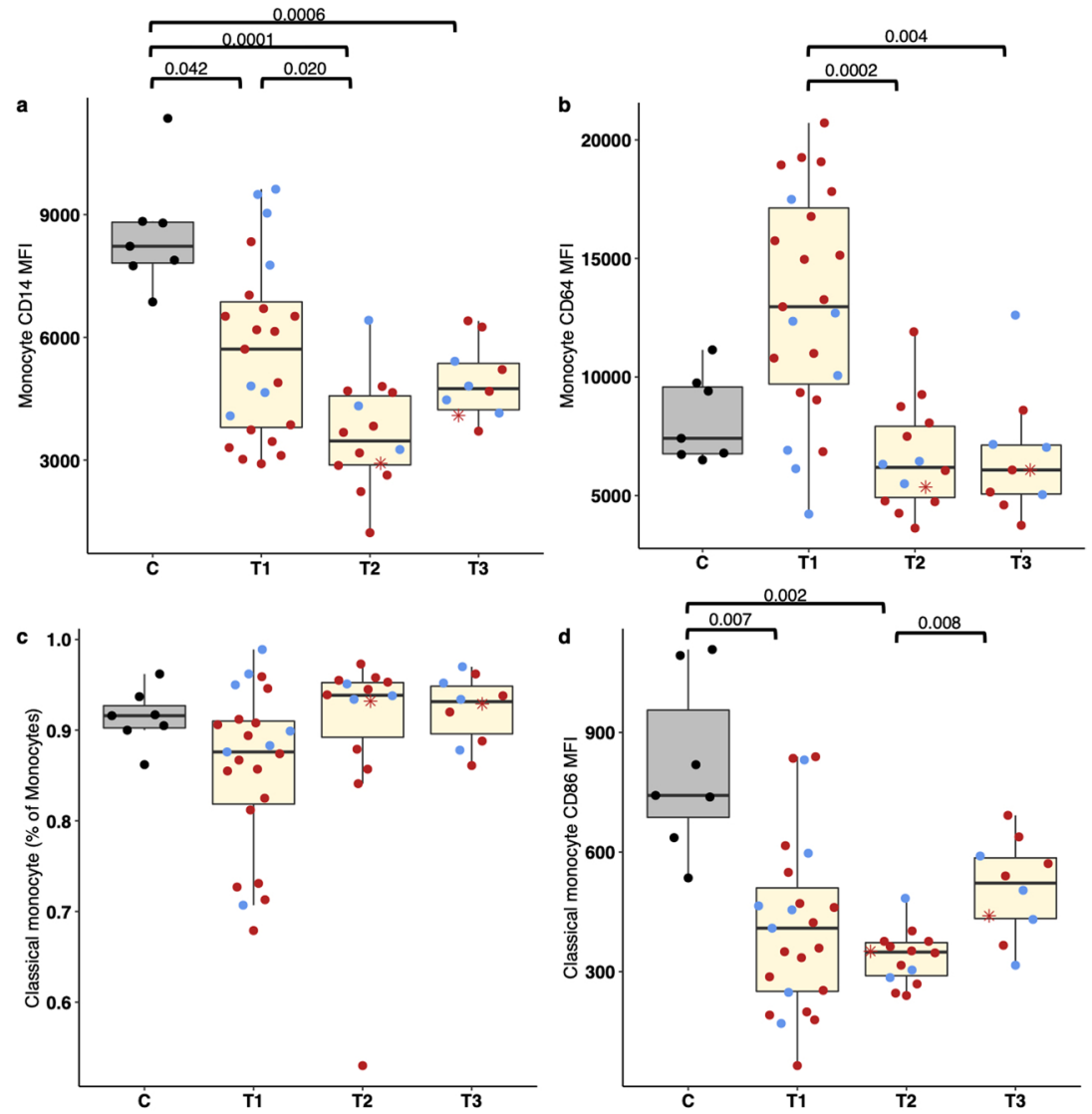

Cohort Control

Extended Data Fig. 4 | Additional information on Innate immune cell alterations in patients with MIS-C at T1, T2 and T3 phases compared to age-matched healthy controls. Innate immune alterations persist in MIS-C. a, Monocyte CD14 (toll-like receptor 4 co-receptor) expression (MFI) was significantly reduced in patients compared with healthy controls. b, Monocyte CD64 (Fc gamma receptor 1) expression (MFI) was increased in acute illness phase (T1) compared with healthy controls and later illness phases (T2 and T3). c, Classical monocyte proportions remained unchanged relative to healthy controls and illness phases. $\mathbf{d}$, Classical monocytes had significantly reduced CD86 ( $\mathrm{T}$ cell co-stimulant) expression (MFI) in acute and resolution illness phases ( 11 and T2) in comparison with healthy controls and convalescence (T3). Abbreviations: MFI, median fluorescence intensity. In figures, box and whisker plots, bottom border represents 25th percentile; line bisecting the shaded region of each box represents median; upper border of the box, 75th percentile. The whiskers represent extreme 1.5 times the 75th (highest) and 25th (lowest) values and markers beyond the extreme line are outliers. Dots are colored by serology result; positive (red) and negative (blue), with the red asterisk indicating the patient positive by RT-PCR for SARS-CoV-2. Healthy Controls are shaded as grey and patients with MIS-C as yellow. Significance testing between patients by phase of illness (T1 ( $n=23$ samples), T2 ( $n=14$ samples), T3 ( $n=10$ samples) was using two-sided Wilcoxon rank sum tests, with correction for multiple comparisons. 

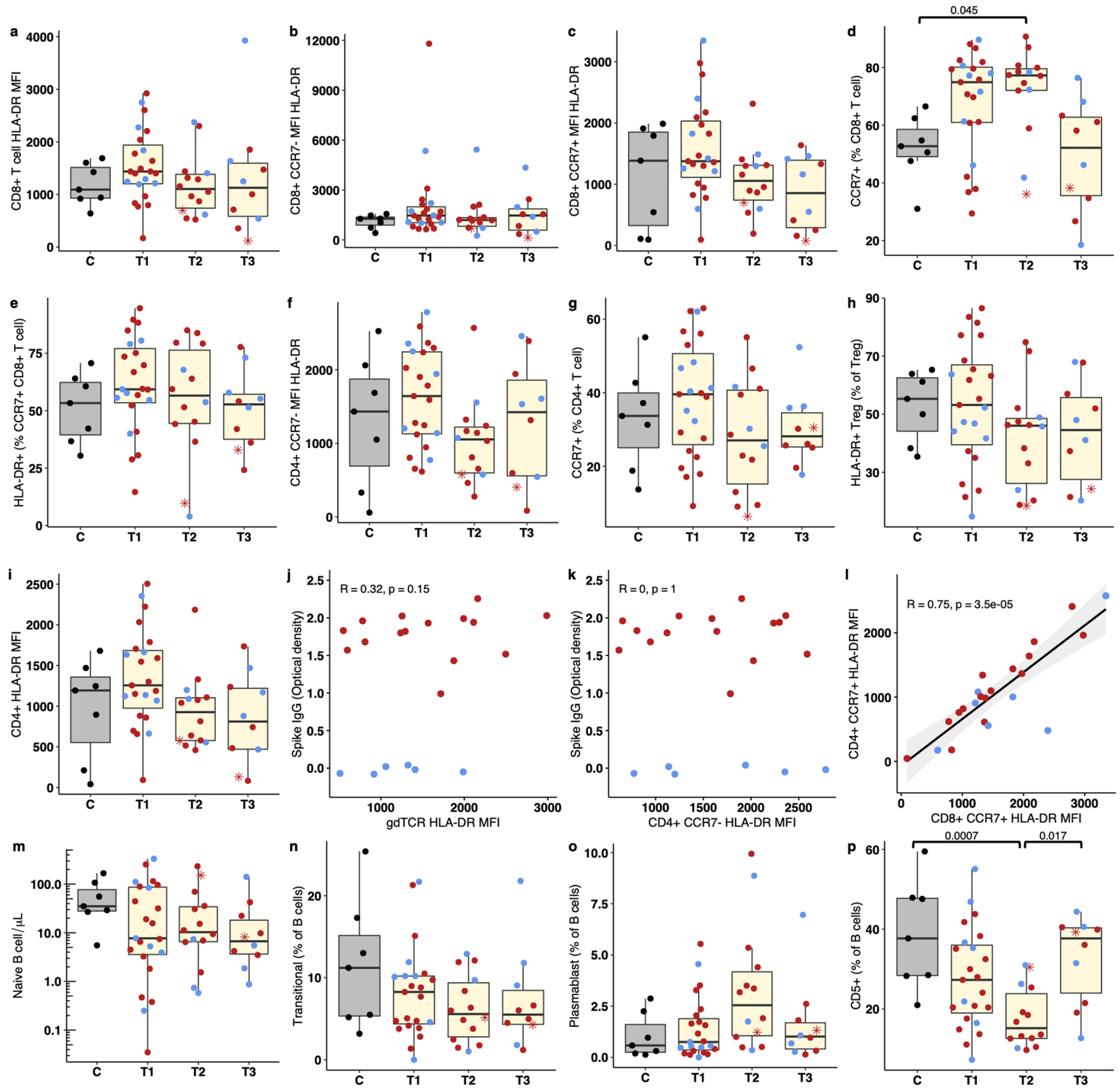

Cohort 由 Control M MIS-C Serology • Control • Negative • Positive SARS-CoV-2 PCR • Negative * Positive

Extended Data Fig. 5 | Additional information on adaptive immune system alterations in patients with MIS-C at T1, T2 and T3 phases compared to age-matched healthy controls. a, HLA-DR expression (MFI) on total CD8+ T cells; $\mathbf{b}, C D 8+C C R 7-T$ cells; and $\mathbf{c}$, CD8+ CCR7+ T cells; $\mathbf{d}$, The percentage of CD8+ CCR7+ T cells; $\mathbf{e}$, The percentage of CD8+ CCR7+ HLA-DR+; f, HLA-DR expression (MFI) on CD4+ CCR7- T cells; $\mathbf{g}$, The percentage of CD4+ CCR7+ T cells; $\mathbf{h}$, The percentage of HLA-RDR+ T regulatory cells (Treg); $\mathbf{i}, \mathrm{HLA}-\mathrm{DR}$ expression (MFI) on total CD4+ T cells; $\mathbf{j}$, HLA-DR MFI on gamma-delta T cells; $\mathbf{k}$, HLA-DR MFI of CD4+ CCR7- T cells was not correlated with IgG to Spike protein; I, HLA-DR MFI of CD4+ CCR7+ and CD8+ CCR7+ T cells was highly correlated; $\mathbf{m}$, Naïve B cell count; $\mathbf{n}$, The percentage of transitional B cells; $\mathbf{0}$, The percentage of plasmablasts; $\mathbf{p}$, The percentage of CD5+ B cells. In figures, box and whisker plots, bottom border represents $25^{\text {th }}$ percentile; line bisecting the shaded region of each box represents median; upper border of the box, $75^{\text {th }}$ percentile. The whiskers represent extreme 1.5 times the $75^{\text {th }}$ (highest) and $25^{\text {th }}$ (lowest) values and markers beyond the extreme line are outliers. Dots are colored by serology result; positive (red) and negative (blue), with the red asterisk indicating the patient positive by RT-PCR for SARS-CoV-2. Healthy Controls are shaded as grey and patients with MIS-C as yellow. Spearman's rank test was used for correlations. Significance testing between patients by phase of illness (T1 ( $n=23$ samples), T2 ( $n=14$ samples), T3 ( $n=10$ samples) was using two-sided Wilcoxon rank sum tests, with correction for multiple comparisons. 
a

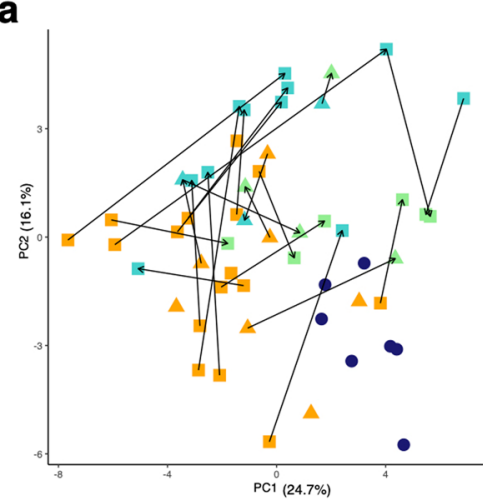

b

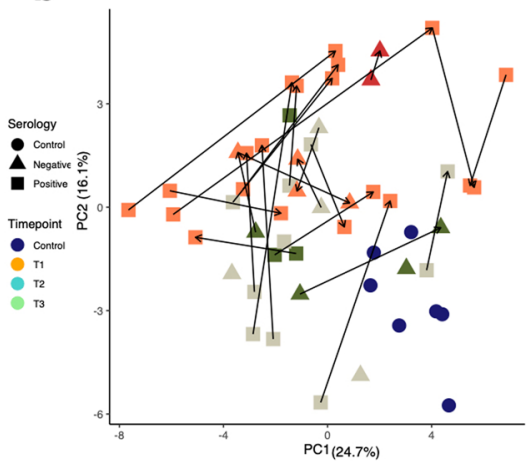

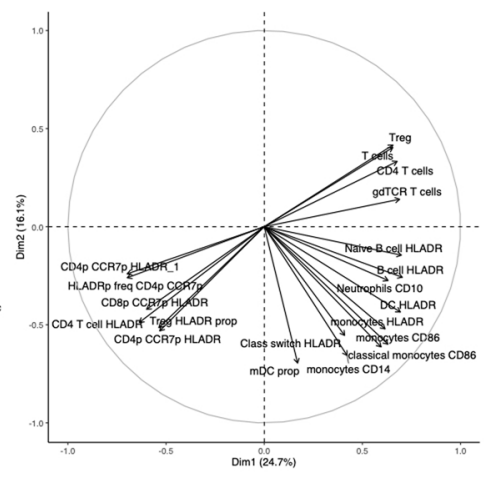

d

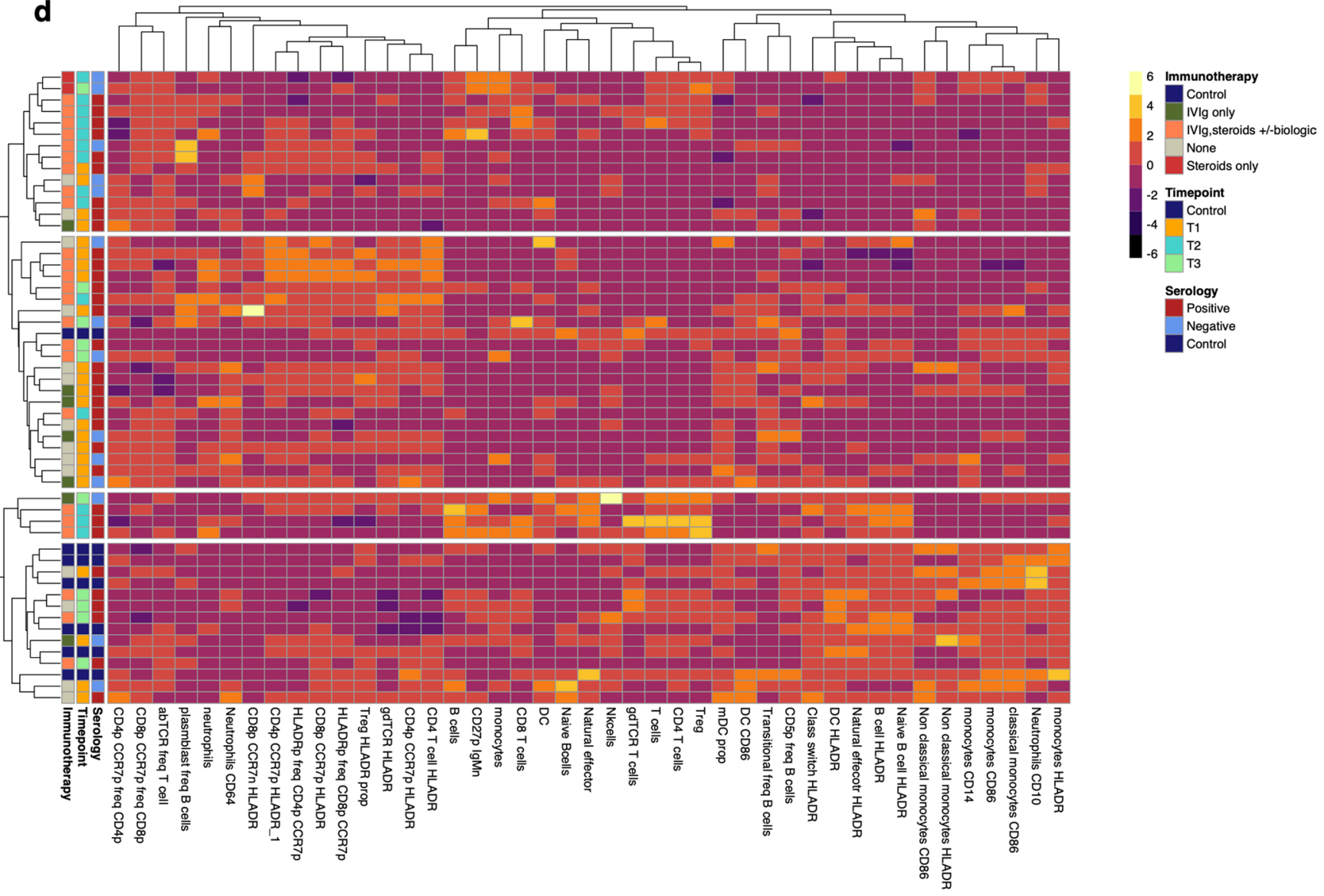

Extended Data Fig. 6 | Unsupervised analysis of flow cytometry immune parameters in patients with MIS-C at T1, T2 and T3 phases compared to age-matched healthy controls. Principal component analysis (PCA) was performed on flow cytometry variables. a, Patient samples are plotted on PC1 and PC2 colored by time point: healthy control, T1 (acute), T2 (resolution), and T3 (convalescent) phases of illness. Shape of point represents SARS-CoV-2 serological status: circle $=$ healthy control, triangle $=$ negative, square $=$ positive. Arrows represent transition of the same patient between serial sampling time points. b, Patient samples are plotted on PC1 and PC2 colored by immunotherapy administered: healthy control, IVIg only, IVIg and steroids+/biologic, none, and steroids only. Arrows represent transition of the same patient between serial sampling time points. c, Contributions of the top 20 variables within the dataset to PC1 and PC2. d, Hierarchal clustering of flow cytometry immune parameters in MIS-C and healthy controls. The left-hand y axes denote immunotherapy administered; time point (T1, T2 or T3) or healthy control; and serological status. The right-hand y axis denotes patient number ( $\mathrm{P}$; as in main Fig. 1a). Abbreviations: IVIg, intravenous immunoglobulin; P. Biologics administered included tocilizumab, infliximab and anakinra (see main Fig. 1a). 


\section{Reporting Summary}

Nature Research wishes to improve the reproducibility of the work that we publish. This form provides structure for consistency and transparency in reporting. For further information on Nature Research policies, see our Editorial Policies and the Editorial Policy Checklist.

\section{Statistics}

For all statistical analyses, confirm that the following items are present in the figure legend, table legend, main text, or Methods section.

$\mathrm{n} / \mathrm{a}$ Confirmed

$\bigotimes$ The exact sample size $(n)$ for each experimental group/condition, given as a discrete number and unit of measurement

$\square$ \ A statement on whether measurements were taken from distinct samples or whether the same sample was measured repeatedly

The statistical test(s) used AND whether they are one- or two-sided

Only common tests should be described solely by name; describe more complex techniques in the Methods section.

$\searrow$ A description of all covariates tested

$\square \bigotimes$ A description of any assumptions or corrections, such as tests of normality and adjustment for multiple comparisons

$\square$ A full description of the statistical parameters including central tendency (e.g. means) or other basic estimates (e.g. regression coefficient)

AND variation (e.g. standard deviation) or associated estimates of uncertainty (e.g. confidence intervals)

For null hypothesis testing, the test statistic (e.g. $F, t, r$ ) with confidence intervals, effect sizes, degrees of freedom and $P$ value noted

$\triangle$ Give $P$ values as exact values whenever suitable.

Х $\square$ For Bayesian analysis, information on the choice of priors and Markov chain Monte Carlo settings

Х $\square$ For hierarchical and complex designs, identification of the appropriate level for tests and full reporting of outcomes

$\square$ \ Estimates of effect sizes (e.g. Cohen's d, Pearson's $r$ ), indicating how they were calculated

Our web collection on statistics for biologists contains articles on many of the points above.

\section{Software and code}

Policy information about availability of computer code

Data collection Flow Cytometry was performed on BD Fortessa using FACS DIVA Software. FCS files were exported and analysed in FlowJo. Statistical analysis and graphing was performed in R Studio using standard functions in Tidyverse and corrplot packages. No custom algorithms were used. 
SOFTWARE:

Flow cytometry: FACS DIVA and FlowJo.

R version 3.6.1 (2019-07-05) -- "Action of the Toes"

Copyright (C) 2019 The R Foundation for Statistical Computing

Platform: x86_64-apple-darwin15.6.0 (64-bit)

RStudio

Version 1.2.1335

R packages:

- tidyverse

- stats

- corrplot

- factoextra

- pheatmap

For manuscripts utilizing custom algorithms or software that are central to the research but not yet described in published literature, software must be made available to editors and reviewers. We strongly encourage code deposition in a community repository (e.g. GitHub). See the Nature Research guidelines for submitting code \& software for further information.

\section{Data}

Policy information about availability of data

All manuscripts must include a data availability statement. This statement should provide the following information, where applicable:

- Accession codes, unique identifiers, or web links for publicly available datasets

- A list of figures that have associated raw data

- A description of any restrictions on data availability

The data presented in this manuscript is provided as a supplementary dataset.

\section{Field-specific reporting}

Please select the one below that is the best fit for your research. If you are not sure, read the appropriate sections before making your selection.

$\bigotimes$ Life sciences

Behavioural \& social sciences

Ecological, evolutionary \& environmental sciences

For a reference copy of the document with all sections, see nature.com/documents/nr-reporting-summary-flat.pdf

\section{Life sciences study design}

All studies must disclose on these points even when the disclosure is negative.

Sample size

Data exclusions

Replication

Randomization

Blinding
This is an exploratory analysis of a novel syndrome and sample size was not predetermined. Due to limited sample size, the statistical significance reported should only be interpreted as indicative of the direction of change in biological signals. However, these data represent the first month of the study at our institution.

Recruitment to the cohort is detailed in eFigure- 1 . With the exception of one child (aged $<6$ months, in comparison to a median cohort age of 12.5 years) all children who were sampled have flow cytometry data presented here. At the time of analysis not all children had completed the time course of disease (detailed as numbers of samples at T1, T2 and T3 in eFigure-1 and eFigure-2). For analysis of serum cytokines, 15 of 25 children had acute serum available prior to administration of intravenous immunoglobulin therapy. Data from these samples are detailed in Figure-1 D.

Standardized sampling, storage and laboratory techniques were used for all patient samples. Batch effects were minimized by the random allocation of samples for processing on sequential days. Due to the limited volume of sample, direct replicates from each sample for flow cytometry was not possible (eMethods 3). For laboratory measurements of immunoglobulin G and M to SARS-CoV-2 using ELISA, measurements were done in duplicate and the mean of the two values was used. For the SARS-CoV-2 pseudotyped virus neutralization assay, measurements were perfromed in duplicate and the duplicates used to calculate ID50. For the cytokine assays using Ella (R\&D Systems) measurements were done in duplicate and the mean of the two values was used. For cytokine assays, three results (on two separate patients) had values beyond the limit of quantitation at standard 1:2 dilution; these samples were repeated at 1:4 dilution.

Participants were not randomized as this was a descriptive cohort study, with the same measurements applied to all patients/samples. The study cohort is described in Table 1 and case definitions in eTable-1. The recruitment of healthy controls is detailed in Methods and described in eTable-2.

This was a descriptive cohort study for which all patients with the clinical syndrome (MIS-C) were eligible following informed (parental if $<16$ years of age) consent. Blinding was therefore not possible for study recruitment. Laboratory measurements (done by MF, AJ, KJD, JS, SA, ET) were made blinded to the clinical status of patients recruited to the study. 


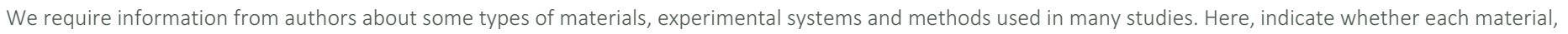

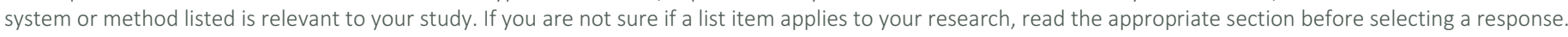

\begin{tabular}{|c|c|c|c|}
\hline \multicolumn{2}{|r|}{ Materials \& experimental systems } & \multicolumn{2}{|c|}{ Methods } \\
\hline $\mathrm{n} / \mathrm{a}$ & Involved in the study & $\mathrm{n} / \mathrm{a}$ & Involved in the study \\
\hline & $\bigotimes$ Antibodies & Х & $\square$ ChIP-seq \\
\hline & Eukaryotic cell lines & & X Flow cytometry \\
\hline Х & Palaeontology and archaeology & Х & MRI-based neuroimaging \\
\hline Х & Animals and other organisms & & \\
\hline & \ Human research participants & & \\
\hline 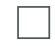 & $\bigotimes$ Clinical data & & \\
\hline Х & Dual use research of concern & & \\
\hline
\end{tabular}

\section{Antibodies}

Antibodies used

Describe all antibodies used in the study; as applicable, provide supplier name, catalog number, clone name, and lot number.

Validation

\section{Eukaryotic cell lines}

Policy information about cell lines

Cell line source(s)

State the source of each cell line used.

Authentication

Describe the authentication procedures for each cell line used OR declare that none of the cell lines used were authenticated.

Mycoplasma contamination

Confirm that all cell lines tested negative for mycoplasma contamination OR describe the results of the testing for

mycoplasma contamination OR declare that the cell lines were not tested for mycoplasma contamination.

Commonly misidentified lines

(See ICLAC register)

Name any commonly misidentified cell lines used in the study and provide a rationale for their use.

\section{Human research participants}

Policy information about studies involving human research participants

Population characteristics

Please see Table 1 in the main manuscript for all population characteristics and relevant co-variates. Timing of treatments (and site of care) is detailed in Figure 1a.

Recruitment

Participants were recruited from the Paediatric Intensive Care and Paediatric Wards at Evelina London Children's Hospital. Recruitment is detailed in Extended Data Figure 1. Recruitment was of consecutive patients with a presumptive diagnosis of MIS-C/PIMS-TS admitted to our institution, following consent and sampling.

Ethics oversight

This descriptive cohort received UK HRA approval (20/HRA/1714) following Research Ethics Committee review (London Dulwich REC). The IRAS ID is 278651.

Note that full information on the approval of the study protocol must also be provided in the manuscript.

\section{Clinical data}

Policy information about clinical studies

All manuscripts should comply with the ICMJE guidelines for publication of clinical research and a completed CONSORT checklist must be included with all submissions.

Clinical trial registration This descriptive cohort received UK HRA approval (20/HRA/1714). The IRAS ID is 278651.

Study protocol

Data collection
The study protocol is the same as the Diamonds Study (https://www.diamonds2020.eu/about/), which was the study to which the patients we report were co-recruited.

The study began recruitment on 28th April 2020, and patients included in this cohort were recruited up until 25th May 2020. Participants were recruited from the Paediatric Intensive Care and Paediatric Wards at Evelina London Children's Hospital. Details of the site of care at each stage of sampling are noted in Figure 1a. Recruitment is detailed in Extended Data Figure 1. 
This is a descriptive cohort study. The pre-defined outcomes of interest were cytokines, serological status, neutralization assay of SARS-CoV-2 antibodies, and major innate and adaptive immune cell subsets. These primary outcomes (i.e. cytokines, serological

\section{Flow Cytometry}

\section{Plots}

Confirm that:

\The axis labels state the marker and fluorochrome used (e.g. CD4-FITC).

$\bigotimes$ The axis scales are clearly visible. Include numbers along axes only for bottom left plot of group (a 'group' is an analysis of identical markers).

$\bigotimes$ All plots are contour plots with outliers or pseudocolor plots.

Х A numerical value for number of cells or percentage (with statistics) is provided.

\section{Methodology}

Sample preparation

Instrument

Software

Cell population abundance

Gating strategy
Whole blood was collected and stored in cytodelics stabilisation buffer at $-80^{\circ} \mathrm{C}$ until analysis. Samples were thawed, stained, fixed and lysed prior to analysis.

Samples were analysed on a BD Fortessa

DIVA was used for sample acquisition on the Fortessa.

FlowJo Version 10.6.2. Becton Dickinson and Company; 2019

Fractions and absolute counts whole blood were measured by flow cytometry analysis. For absolute counts Biolegend Precision count beads were added prior to acquisition.

Gating strategies for all panels are shown in the supplementary material.

Х Tick this box to confirm that a figure exemplifying the gating strategy is provided in the Supplementary Information. 